OPEN ACCESS

Edited by:

Rajeev K. Singla,

Netaji Subhas Institute of Technology,

India

Reviewed by:

Dharmendra Kumar Yadav, Gachon University of Medicine and

Science, South Korea

Andrea Gualandi,

Università di Bologna, Italy

*Correspondence:

Abdul Sadic

sadiquom@yahoo.com

Umer Rashid

umerrashid@ciit.net.pk

Specialty section:

This article was submitted to

Medicinal and Pharmaceutical

Chemistry,

a section of the journa

Frontiers in Chemistry

Received: 18 September 2017

Accepted: 22 February 2018

Published: 12 March 2018

Citation

Jabeen M, Ahmad S, Shahid K Sadiq A and Rashid U (2018) Ursolic Acid Hydrazide Based Organometallic

Complexes: Synthesis,

Characterization, Antibacterial,

Antioxidant, and Docking Studies.

Front. Chem. 6:55.

doi: 10.3389/fchem.2018.00055

\section{Ursolic Acid Hydrazide Based Organometallic Complexes: Synthesis, Characterization, Antibacterial, Antioxidant, and Docking Studies}

\author{
Muafia Jabeen ${ }^{1}$, Sajjad Ahmad ${ }^{2}$, Khadija Shahid ${ }^{1}$, Abdul Sadiq $^{2 *}$ and Umer Rashid ${ }^{3 *}$ \\ ' Department of Pharmaceutical Chemistry, Riphah Institute of Pharmaceutical Sciences, Riphah International University, \\ Islamabad, Pakistan, ${ }^{2}$ Department of Pharmacy, University of Malakand, Chakdara, Pakistan, ${ }^{3}$ Department of Chemistry, \\ COMSATS Institute of Information Technology, Abbottabad, Pakistan
}

In thecurrent research work, eleven metal complexes were synthesized from the hydrazide derivative of ursolic acid. Metal complexes of tin, antimony and iron were synthesized and characterized by FT-IR and NMR spectroscopy. The antibacterial and antioxidant activities were performed for these complexes, which revealed that the metal complexes synthesized are more potent than their parent compounds. We observed that antioxidant activity showed by triphenyltin complex was significant and least activity have been shown by antimony trichloride complex. The synthesized metal complexes were then evaluated against two Gram-negative and two Gram-positive bacterial strains. Triphenyl tin complex emerged as potent antibacterial agent with MIC value of $8 \mu \mathrm{g} / \mathrm{ml}$ each against Shigellaspp, Salmonella typhi and Staphylococcus aureus. While, the MIC value against Streptococcus pneumoniae is $4 \mu \mathrm{g} / \mathrm{ml}$. Computational docking studies were carried out on molecular targets to interpret the results of antioxidant and antibacterial activities. Based on the results, it may be inferred that the metal complexes of ursolic acid are more active as compared to the parent drug and may be proved for some other pharmacological potential by further analysis.

Keywords: ursolic acid, triterpenoid, metal complex, hydrazine, antioxidant, antibacterial, molecular docking

\section{INTRODUCTION}

Ursolic acid (3ß-hydroxy-urs-12-ene-28-oic acid, UA) is a triterpenoid present in many medicinal plants, such as Eriobotrya japonica, Rosmarinus officinalis, Ocimum sanctum, Melaleuca leucadendron, Piper betle, and Glechomahederaceae (Liu, 1995). Ursolic acid has been reported with excellent biological potential. It exerts anti-inflammatoryeffects by inhibiting arachidonate metabolism (Baricevic et al., 2001) and also increasesthe nitric oxide synthase activity in endothelial cells (Shin et al., 2004). UA acts as an inhibitor of PTP1b with 10-times greater potency, topromote insulin receptor phosphorylation and glucose uptake in L6 myotubes (Gum et al., 2003). Ithas strong hepatoprotective activity against ethanol. Balanehru et al have shown that UA has strong protective effect against free radical damage in heart than in liver in-vitro condition (Balanehru and Nagarajan, 1992). It also play a vital role in suppressionofaberrant crypt foci (ACF) formation 
and have been noted to be protective against colon carcinogenesis (Furtado et al., 2008). Ursolic acid and its derivatives have been reported with antibacterial, antioxidant, anticancer and anti-ulcer potentials by different research groups (Farina et al., 1998; Wolska et al., 2010; do Nascimento et al., 2014; Kalani et al., 2014). Hydrazine, hydrazone and its other analogs are potential group of compounds for various pharmacological potentials. Being a reactive functionality, the acid derivatives of hydrazine are widely synthesized (Audrieth et al., 1954). The most prominent activity reported with hydrazine and its<smiles>CC(O)CO</smiles><smiles>CCOC(=O)C12CCC(C)C(C)C1C1=CCC3C(C)(CCC4C(C)(C)C(O)CCC43C)C1CC2</smiles>

Ethylester of ursolic acid<smiles>CC1CCC2(C(=O)NN)CC[C@H]3C(=CCC4C5(C)CCC(O)C(C)C5CCC43C)C2C1C</smiles>

FIGURE 1 | Synthesis of Ligand.

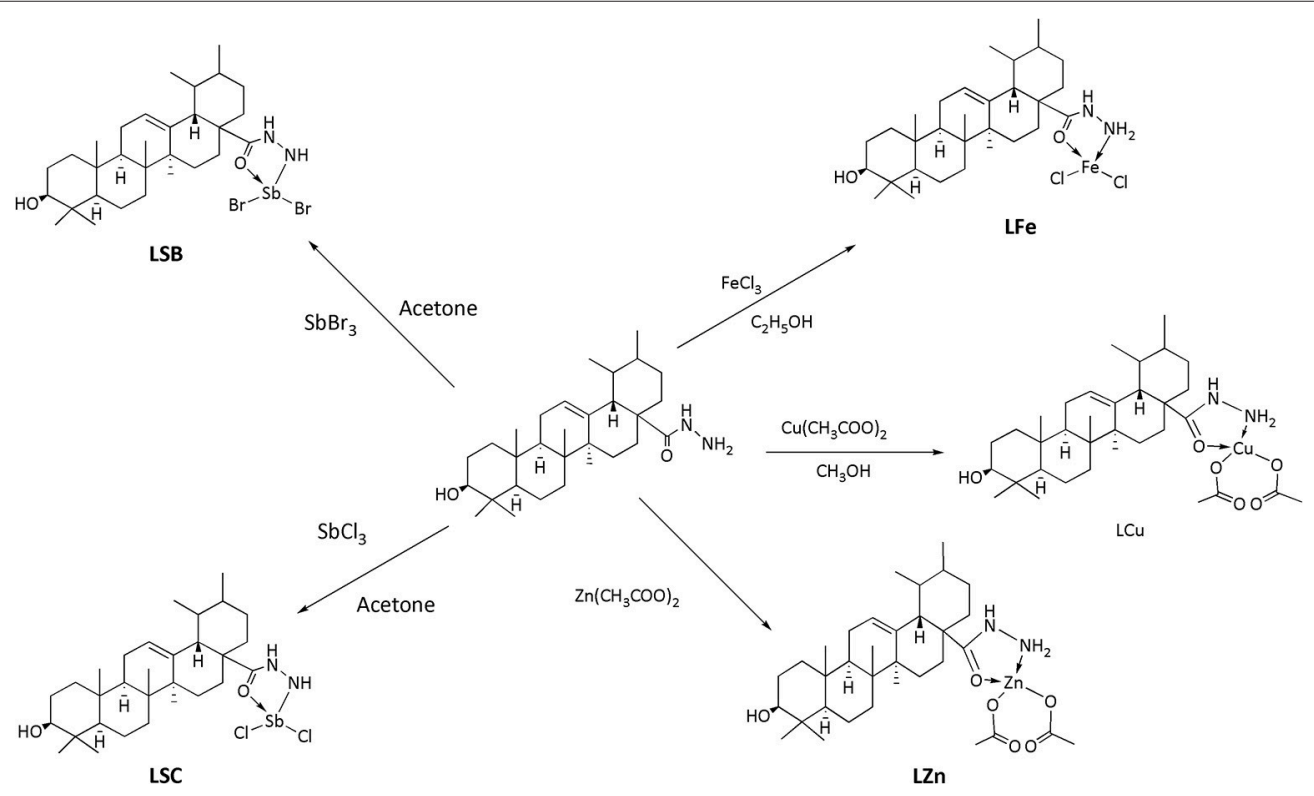

FIGURE 2 | Synthesis of $\mathrm{Cu}, \mathrm{Fe}, \mathrm{Zn}$ and $\mathrm{Sb}$ complexes. 
related compounds is the free radicals scavenging effect (Gürkök et al., 2009; Belkheiri et al., 2010; Zhong et al., 2010; Musad et al., 2011; Yilmaz et al., 2012). However, various researchers have also explored the antibacterial and antifungal potentials of such nitrogenous compounds against various microbial strains (Parodi et al., 1981; Akbas and Berber, 2005; Sönmez et al., 2006; Salimon et al., 2010). The hydrazine derivatives have also potential effect as a chemotherapeutic agent for the management of Tuberculosis (Robitzek et al., 1952; Selikoff et al., 1952). Like many other synthetic and natural bioactive compounds (Sadiq et al., 2015; Shah et al., 2015; Zeb et al., 2017), UA has been included in important medicinal compounds and investigational studies are being carried out in various aspects (Ziegler et al., 2004; Liu, 2005).

The metal complexesplay vital roles in catalysis, materials synthesis and photochemistry (Greenwood and Earnshaw, 2012). Tin, a metallic element strongly affects the biochemical property of organo-tin compounds (Jastrzebski and Van Koten, 1993). Therefore,organo-tin (IV) complexes, organo-tin (IV) carboxylates have potent antifungal, antibacterial, and antitumor activities (Davies and Smith, 1980). Commonly, triorgano-tin (IV) compounds are more active than their di and monoorganotin (IV) analogs, this activity related to binding of proteins (Blunden and Evans, 1990). Copper (Cu) hasanticancer activity, particularly used against breast cancer cells and proliferative cells (MCF7) (Yousefi et al., 2011). CopperII complexes display cytotoxic activity against human acute lymphoblastic leukemia CCRF-CEM cells and colon adenocarcinoma HT-29 cells an also used as antiseptic (Easmon et al., 2001). Copper is involved in hemoglobin formation, drug/xenobiotic metabolism, carbohydrate metabolism, catecholamine biosynthesis and in cross-linking of collagen, elastin and hair keratin as well as in the antioxidant defense characteristics because it is present in many metallo-enzyme (Ergene et al., 2010). Similarly, Hydrazine (diazane) is an inorganic compound with the formula $\mathrm{N}_{2} \mathrm{H}_{4}$, possessing $\mathrm{H}_{2} \mathrm{~N}-\mathrm{NH}$ - subunit and constitute an important class of compounds for new drug development. Hydrazides are the derivatives of hydrazine with at least one acyl group [R$\mathrm{C}(=\mathrm{O})-\mathrm{NH}-\mathrm{NH}_{2}$ ]. Medicinal and drug discovery chemists are in constant research to synthesize these compounds as target structures and to embark their various biological potentials (Kim et al., 1999).

In this research work, hydrazine derivative of ursolic acid was synthesized as a ligand and then different metal complexes of the ligand were synthesized. Furthermore, the antibacterial and antioxidant activities of the complexes were carried out. Additionally, we have supported our in-vitro results with in-silico studies.

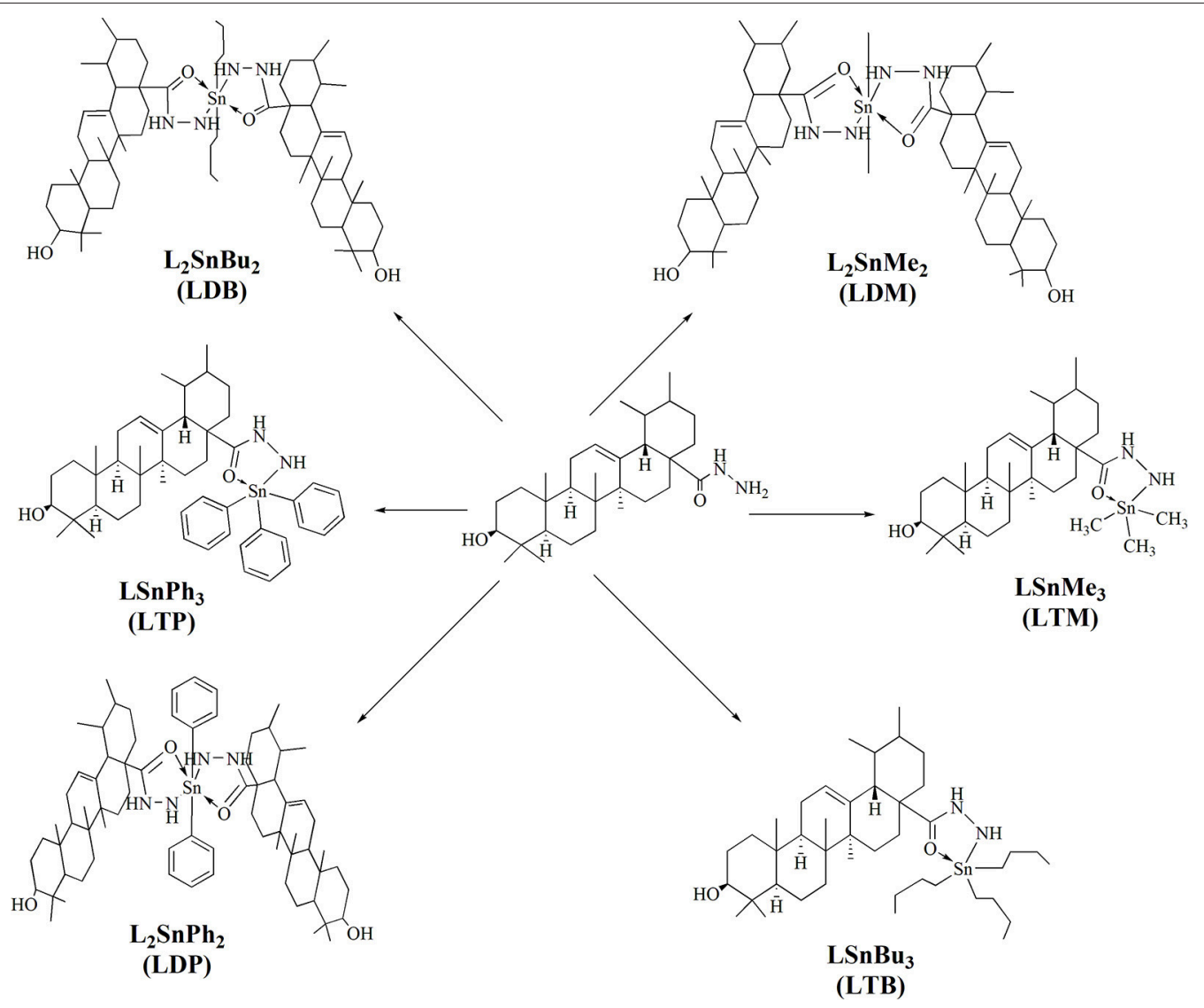

FIGURE 3 | Synthesis of tin complexes. 


\section{MATERIALS AND METHODS}

\section{Synthesis of Ligand}

Direct etherification method was used for the synthesis of esters. In a flask, mixture containing 0.2 moles of UA, conc. sulphuric acid $(0.4 \mathrm{~mol})$ and $0.2 \mathrm{ml}$ of ethanol were taken and the mixture was allowed to reflux for $3 \mathrm{~h}$. Surplus ethanol was distilled off under low pressure employing rotary apparatus and allowed the mixture to cool at room temperature. It was then filtered and the residue obtained was dried over silica gel in a desiccator.

Hydrazine hydrate $(0.1 \mathrm{~g})$ and ethyl ester of UA (0.002 mol) were taken in a flask. A small volume of ethanol was mixed for the formation of clear reaction mixture. For $3-5 \mathrm{~h}$ the contentwas allowed to reflux. The evaporation of excess ethanol was carried out via rotary evaporator to get the productin $85 \%$ yield.

\section{Synthesis of Metal Complexes}

Procedure for the Synthesis of Copper Complex (LCu) Copper complex was synthesized by using $2.0 \mathrm{mmol}$ of hydrazide of UA (ligand, UL) in $30 \mathrm{~mL}$ of (solution 1). In another flask, copper acetate monohydrate $\left[\mathrm{Cu}\left(\mathrm{CH}_{3} \mathrm{COO}\right)_{2} \cdot \mathrm{H}_{2} \mathrm{O}, 2.0 \mathrm{mmol}\right]$ was mixed in $20 \mathrm{~mL}$ of methanol (solution 2). Solution 1 was added with solution 2 . The reaction mixture was maintained at $\mathrm{pH}$ of $7.0 \pm 0.05$ with the help of triethylamine. The mixture was stirred for $4 \mathrm{~h}$ at room temperature and resultant mixture was allowed to stay overnight in refrigerator. A green colored precipitates was filtered by filtration, and dried over silica gel in a desiccator. The pure product was obtained in $60 \%$ yield.

\section{Procedure for the Synthesis of Copper Complex (LZn)} Ligand (UL, $1.0 \mathrm{mmol}$ ) and zinc acetate dihydrate $\left[\mathrm{Zn}(\mathrm{CH} 3 \mathrm{COOH})_{2} .2 \mathrm{H}_{2} \mathrm{O}, 1.0 \mathrm{mmol}\right]$ were mixed in equal volume of methanol $(25 \mathrm{ml})$ separately, after mixing $\mathrm{pH}$ of solution was maintained at 7.5 with the addition of $0.1 \%$ potassium hydroxide in methanol. The mixture was then refluxed for $3 \mathrm{~h}$. Finally, the mixture was filtered and dried over silica gel to obtain a light yellow product in $65 \%$ yield.

TABLE 1 | Physical data of ligand and their metal complexes.

\begin{tabular}{|c|c|c|c|c|c|}
\hline S. $\mathbf{N}$ & $\begin{array}{l}\text { Identity } \\
\text { codes }\end{array}$ & Mol. Formula & $\begin{array}{c}\text { Formula } \\
\text { weight (g/mol) }\end{array}$ & $\begin{array}{l}\text { Isolated } \\
\text { yield (\%) }\end{array}$ & $\begin{array}{l}\text { Melting point } \\
\left({ }^{\circ} \mathrm{C}\right) \& \text { color }\end{array}$ \\
\hline 1 & UL & Ligand & 471 & 85 & 115, White \\
\hline 2 & LCu & $\mathrm{LCu}\left(\mathrm{CH}_{3} \mathrm{COO}\right)_{2}$ & 652 & 60 & 80, light Gray \\
\hline 3 & $\mathrm{LZn}$ & $\mathrm{LZn}\left(\mathrm{CH}_{3} \mathrm{COO}\right)_{2}$ & 690 & 65 & 155, White \\
\hline 4 & LFe & $\mathrm{LFeCl}_{2}$ & 597 & 65 & 160, off White \\
\hline 5 & LSC & $\mathrm{LSbCl}_{2}$ & 663 & 73 & 180, off White \\
\hline 6 & LSB & LSbBr2 & 752 & 72 & 170, off White \\
\hline 7 & LDM & $\mathrm{LMe}_{2} \mathrm{SnCl}_{2}$ & 690 & 65 & 100, off White \\
\hline 8 & LTM & $\mathrm{LMe}_{3} \mathrm{SnCl}$ & 670 & 67 & 80, Yellowish \\
\hline 9 & LDB & $\mathrm{LBu}_{2} \mathrm{SnCl}_{2}$ & 774 & 70 & 95, White \\
\hline 10 & LTB & $\mathrm{LBu}_{3} \mathrm{SnCL}$ & 796 & 72 & 130, Yellowish \\
\hline 11 & LDP & $\mathrm{LPhe}_{2} \mathrm{SnCL}_{2}$ & 815 & 60 & 140, Yellowish \\
\hline 12 & LTP & $\mathrm{LPhe}_{3} \mathrm{SnCl}$ & 856 & 65 & 180, Grayish \\
\hline
\end{tabular}

Procedure for the Synthesis of Iron Complex (LFe)

A solution of Ligand $(1.0 \mathrm{mmol})$ in ethanol $(5.0 \mathrm{ml})$ was mixed and then solution of $\mathrm{FeCl}_{3}(1.0 \mathrm{mmol})$ was added to it. The mixture was refluxedfor $50 \mathrm{~min}$ with continuous stirring. The obtainedcolored solution was reserved at room temperature. In the filtrate, the obtained product was dried with silica gel in a desiccator. The pure product was obtained in $65 \%$ yield.

\section{Procedure for the Synthesis of Antimony Complex (LSC and LSB) by Using Antimony Trichloride/Bromide}

Ligand $(1.0 \mathrm{mmol})$ in $15 \mathrm{ml}$ of acetonitrile and solution of $\mathrm{SbCl}_{3} /$ $\operatorname{SbBr}_{3}(1.0 \mathrm{mmol})$ in $15 \mathrm{ml}$ of acetone was added according to molar ratio of $1: 1$. The mixture was stirred for $30 \mathrm{~min}$. The mixture was filtered and allowed to set for crystallization at room temperature. The desire product was dried in silica gel desiccator. The pure product was obtained in $73 \%$ yield (from $\mathrm{SbCl}_{3}$ ) and $72 \%$ (from $\mathrm{SbBr}_{3}$ ).

\section{Procedure for the Synthesis of Tin Complexes (LDM, LTM, LDB, LTB, LDP, LTP)}

A ligand's solution $(1.0 \mathrm{mmol})$ was prepared in $50 \mathrm{ml}$ of toluene (dry). Then triethylamine $(1.0 \mathrm{mmol})$ was added to the ligand solution and refluxed for $3 \mathrm{~h}$. After-wards, the reaction mixture was cooled down to room temperature. Organotin compounds $(1.0 \mathrm{mmol})$ was added to a flask with continuous stirring and refluxed again for 8-10 h. After reflux completion, the mixture was filtered. The solvent was evaporated through rotary evaporator. The residue was obtained and re-crystallized with the help of chloroform (Atta-ur-Rahman et al., 2001). Dimethyltin complex (LDM) was obtained in $65 \%$ yield. While, trimethyl complex (LTM), dibutyl (LDB), tributyl (LTB), diphenyl (LDP) and triphenyltin complex (LTP) were obtained in $67,70,72,60$, and $65 \%$ yield respectively.
TABLE 2 | Solubility of the ligand and its metal complexes.

\begin{tabular}{lllllll} 
S. N & Compound & Methanol & Ethanol & DMSO* & Chloroform & Toluene \\
\hline 1 & UL & Yes* & No & Yes & Yes & Yes \\
2 & LZn & No & No & Yes & Yes & Yes \\
3 & LCu & No & Yes & Yes & No & Yes \\
4 & LSB & Yes & Yes & Yes & Yes & Yes \\
5 & LSC & Yes & Yes & No & No & No \\
6 & LFe & Yes & Yes & Yes & Yes & Yes \\
7 & LTP & No & No & Yes & Yes & Yes \\
8 & LDP & Yes & Yes & No & No & No \\
9 & LTB & Yes & Yes & No & No & No \\
10 & LDB & Yes & Yes & No & No & No \\
11 & LTM & Yes & Yes & No & No & No \\
12 & LDM & Yes & Yes & Yes & Yes & Yes \\
\hline${ }^{*}$ Dimethylsulfoxide. & & & & &
\end{tabular}




\section{Solubility Method}

First of all apparent solubility of all the synthesized metals were determined. A weighed amount of the metal complexes were dissolved in measured amount of solvent and clarity/turbidity was observed. Another experimentally simple method for the solubility determination was carried out as described by Harle et al. (2003) using Buchner funnel. Methanol, ethanol, DMSO, chloroform and toluene were used for this study. For good solubility, the threshold value of $<0.1 / 100 \mathrm{~mL}$ with a clear colored solution in the given solvent was considered.

\section{Antibacterial Assay}

The antibacterial activity of ursolic acid, the ligand and their metal complexes were determined against various bacterial strains using well diffusion assay. The tested strains include Gram negative like, Escherichia coli, Salmonella typhi, and Shigella species, and Gram positive strains include Staphylococcus aureusand Bacillus subtilus.

Aliquot of nutrient broth $(10 \mathrm{ml})$ was made in this method which was inoculated with bacteria at $37( \pm 1)^{\circ} \mathrm{C}$ for $24 \mathrm{~h}$. Broth culture $(0.6 \mathrm{ml})$ of the test organism was added in a Petri dish via a sterile pipette. This was also included with $20 \mathrm{ml}$ of agar having $0.2 \mathrm{ml}$ culture and was heated at $45^{\circ} \mathrm{C}$. This was suspended properly and was added into a sterile Petri dish. Plate of each organism was obtained in a similar way. The agar was allowed to dry/solidify and with the help of a sterile cork borer, the required numbers of holes $(10 \mathrm{~mm})$ were cut. The holes were designed and distributed properly, one in the center and others in the periphery. The agar plugs were removed.

Solution of the ligand and metal complexes were made individually having concentration of $1.0 \mathrm{mg} / \mathrm{ml}$. With the help of micropipette, test sample of $100 \mu \mathrm{l}$ was added in a solvent. The holes were labeled and the test samples were added properly. A solution of tetracycline $(1.0 \mathrm{mg} / \mathrm{ml})$ was used as positive control. The plates are allowed to stay at room temperature for $2 \mathrm{~h}$ for proper diffusion of the sample and incubated at $37 \pm 1^{\circ} \mathrm{C}$ for $24 \mathrm{~h}$. Hence antibacterial activity was determined by calculations of the zone of inhibition in millimeters (Sadiq et al., 2016).

\section{Determination of Antifungal Activity by Agar Tube Dilution Assay}

The antifungal activity of UA, ligand and their metal complexes were evaluated against six (6) fungal strains. The fungal

TABLE 3 | IR spectral analysis of ligand and its metal complexes.

\begin{tabular}{|c|c|c|c|c|c|c|c|}
\hline \multirow[t]{2}{*}{ Complex } & \multicolumn{7}{|c|}{ Frequency $\left(\mathrm{cm}^{-1}\right)$} \\
\hline & $\mathrm{C}=\mathrm{C}$ & $\mathrm{C}=\mathrm{O}$ & $\mathrm{NH}_{2}$ & $\tilde{\mathbf{N H}}$ & $\tilde{\mathbf{N M}}$ & $\tilde{\mathbf{M O}}$ & $\tilde{\text { cos }}$ \\
\hline Ligand(UL) & 1,650 & 1,508 & $3,358^{s}, 1,637^{b}$ & 3,209 & - & - & 1,215 \\
\hline LTP & 1,680 & 1,508 & 3,360 & 3,197 & 570 & 435 & 1,047 \\
\hline LDP & 1,695 & 1,508 & $3,253^{s}, 1,635^{b}$ & 3,151 & 580 & 483 & 1,031 \\
\hline LTB & 1,680 & 1,508 & $3,365^{s}$ & 3209 & 501 & 401 & 1,211 \\
\hline LDB & 1,650 & 1,550 & $1,597^{\mathrm{b}}$ & 2,951 & 513 & 410 & 1,031 \\
\hline LTM & 1658 & 1561 & $1608^{b}$ & 3,034 & 545 & 651 & 1029 \\
\hline LDM & 1,658 & 1,591 & $1,614^{b}$ & $3,240^{s}$ & 540 & - & 1033 \\
\hline LFe & 1,633 & 1,506 & $1,633^{\mathrm{b}}$ & $3,209^{1}$ & 511 & 401 & 1,109 \\
\hline $\mathrm{LCuSO}_{4}$ & 1,650 & 1,508 & $3,354^{s}, 1,602^{b}$ & - & 495 & 414 & 1,012 \\
\hline $\mathrm{L} Z \mathrm{n}(\mathrm{Ac})_{2}$ & 1625 & 1539 & 3348 & 3,257 & 491 & 400 & 1,012 \\
\hline $\mathrm{LSbCl}_{2}$ & 1,650 & 1,510 & $3,360^{s}, 1,633^{b}$ & 3,273 & 567 & 416 & 1,049 \\
\hline $\mathrm{LSbBr}_{2}$ & 1,680 & 1,513 & $3,367^{s}$ & - & 578 & 433 & 1,064 \\
\hline
\end{tabular}

s Stretching frequency.

${ }^{b}$ Bending frequency.

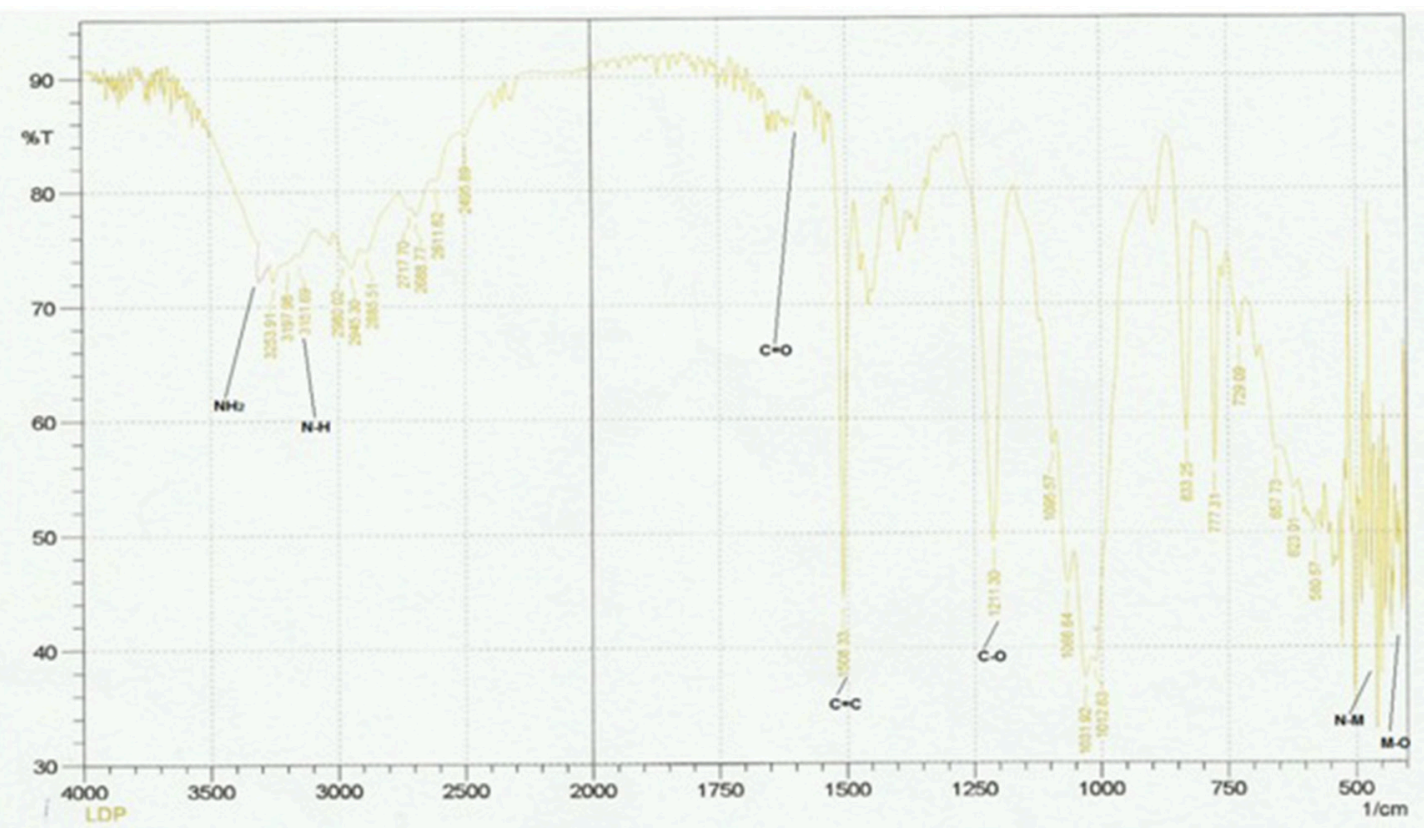

FIGURE 4 | IR spectrum of diphenyltin complex. 
strains used wereCandida alibican, Tricophytonlongifusus, Microsporumcanis, Asperigillusflavus, Fusarium solani, and Bipol using agar tube diffusion method. Sabouraud 4\% Glucose Agar was mixed in distilled water with stirring to make sabouraud dextrose agar and heated. Prepared media was added into test tubes and autoclaved at for $15 \mathrm{~min}$ at $121^{\circ} \mathrm{C}$. Autoclaved

TABLE 4A | ${ }^{1} \mathrm{HNMR}$ spectral data of ligand and their metal complexes.

\begin{tabular}{|c|c|c|c|c|c|c|c|}
\hline $\begin{array}{l}\text { H } \\
\text { No }\end{array}$ & $\begin{array}{l}\text { Compound } \\
\text { assignment }\end{array}$ & $\begin{array}{c}\mathbf{L} \\
\delta(\mathrm{ppm})\end{array}$ & $\begin{array}{l}\mathrm{LFeCl}_{2} \\
\delta(\mathrm{ppm})\end{array}$ & $\begin{array}{c}\mathrm{LCu}(\mathrm{Ac})_{2} \\
\delta(\mathrm{ppm})\end{array}$ & $\begin{array}{c}\mathrm{LZn}(\mathbf{A c})_{2} \\
\delta(\mathrm{ppm})\end{array}$ & $\begin{array}{l}\mathrm{LSbCl}_{3} \\
\delta(\mathrm{ppm})\end{array}$ & $\begin{array}{l}\mathrm{LSbBr}_{3} \\
\delta(\mathrm{ppm})\end{array}$ \\
\hline 1 & $\begin{array}{l}\text { a.CH } \\
\text { b. } \mathrm{CH}\end{array}$ & $\begin{array}{l}1.70 \\
1.28\end{array}$ & $\begin{array}{l}1.25 \\
1.47\end{array}$ & $\begin{array}{l}1.70 \\
1.25\end{array}$ & $\begin{array}{l}1.45 \\
1.25\end{array}$ & $\begin{array}{l}1.20 \\
1.30\end{array}$ & $\begin{array}{l}1.23 \\
1.35\end{array}$ \\
\hline 2 & $\begin{array}{l}\text { a.CH } \\
\text { b. } \mathrm{CH}\end{array}$ & $\begin{array}{l}1.71 \\
1.25\end{array}$ & $\begin{array}{l}1.71 \\
1.47\end{array}$ & $\begin{array}{l}1.71 \\
1.38\end{array}$ & $\begin{array}{l}1.72 \\
1.45\end{array}$ & $\begin{array}{l}1.40 \\
1.70\end{array}$ & $\begin{array}{l}1.43 \\
1.71\end{array}$ \\
\hline 3 & $\mathrm{CH}$ & 3.11 & 3.15 & 3.30 & 3.16 & 3.15 & 3.17 \\
\hline $3^{\prime}$ & $\mathrm{OH}$ & 2.40 & 2.25 & 2.50 & 2.50 & 2.10 & 2.20 \\
\hline 4 & - & - & - & - & - & - & - \\
\hline 5 & $\mathrm{CH}$ & 1.29 & 1.35 & 1.37 & 1.38 & 1.31 & 1.32 \\
\hline 6 & $\begin{array}{l}\text { a.CH } \\
\text { b. } \mathrm{CH}\end{array}$ & $\begin{array}{l}1.27 \\
1.73\end{array}$ & $\begin{array}{l}1.52 \\
1.27\end{array}$ & $\begin{array}{l}1.23 \\
1.50\end{array}$ & $\begin{array}{l}1.50 \\
1.25\end{array}$ & $\begin{array}{l}1.42 \\
1.27\end{array}$ & $\begin{array}{l}1.43 \\
1.27\end{array}$ \\
\hline 7 & $\begin{array}{l}\text { a.CH } \\
\text { b.CH }\end{array}$ & $\begin{array}{l}1.63 \\
1.16\end{array}$ & $\begin{array}{l}1.47 \\
1.24\end{array}$ & $\begin{array}{l}1.48 \\
1.25\end{array}$ & $\begin{array}{l}1.50 \\
1.24\end{array}$ & $\begin{array}{l}1.28 \\
1.47\end{array}$ & $\begin{array}{l}1.27 \\
1.46\end{array}$ \\
\hline 8 & - & - & - & - & - & - & - \\
\hline 9 & $\mathrm{CH}$ & 1.57 & 1.43 & 1.43 & 1.50 & 1.43 & 1.41 \\
\hline 10 & - & - & - & - & - & - & - \\
\hline 11 & $\begin{array}{l}\text { a. } \mathrm{CH} \\
\text { b. } \mathrm{CH}\end{array}$ & $\begin{array}{l}2.20 \\
1.27\end{array}$ & $\begin{array}{l}2.01 \\
1.16\end{array}$ & $\begin{array}{l}2.10 \\
1.75\end{array}$ & $\begin{array}{l}1.88 \\
1.76\end{array}$ & $\begin{array}{l}1.70 \\
2.04\end{array}$ & $\begin{array}{l}1.80 \\
2.13\end{array}$ \\
\hline 12 & $\mathrm{CH}$ & 4.70 & 5.28 & 5.21 & 5.11 & 4.60 & 4.70 \\
\hline 13 & - & - & - & - & - & - & - \\
\hline 14 & - & - & - & - & - & - & - \\
\hline 15 & $\begin{array}{l}\text { a.CH } \\
\text { b. } \mathrm{CH}\end{array}$ & $\begin{array}{l}1.27 \\
1.11\end{array}$ & $\begin{array}{l}1.31 \\
1.16\end{array}$ & $\begin{array}{l}1.29 \\
1.13\end{array}$ & $\begin{array}{l}1.30 \\
1.16\end{array}$ & $\begin{array}{l}1.10 \\
1.14\end{array}$ & $\begin{array}{l}1.12 \\
1.13\end{array}$ \\
\hline 16 & $\begin{array}{l}\text { a. } \mathrm{CH} \\
\text { b.CH }\end{array}$ & $\begin{array}{l}1.25 \\
1.11\end{array}$ & $\begin{array}{l}1.62 \\
1.36\end{array}$ & $\begin{array}{l}1.61 \\
1.35\end{array}$ & $\begin{array}{l}1.50 \\
1.26\end{array}$ & $\begin{array}{l}1.70 \\
1.31\end{array}$ & $\begin{array}{l}1.71 \\
1.31\end{array}$ \\
\hline 17 & - & - & - & - & - & - & - \\
\hline 18 & $\mathrm{CH}$ & 2.49 & 2.30 & 2.30 & 2.50 & 2.50 & 2.76 \\
\hline 19 & $\begin{array}{l}\text { a. } \mathrm{CH} \\
\text { b. } \mathrm{CH}\end{array}$ & 1.71 & 1.63 & 1.53 & 1.76 & 1.70 & 1.65 \\
\hline 20 & - & - & 1.61 & 1.45 & 1.56 & 1.60 & 1.65 \\
\hline 21 & $\begin{array}{l}\text { a. } \mathrm{CH} \\
\text { b. } \mathrm{CH}\end{array}$ & $\begin{array}{l}1.39 \\
1.16\end{array}$ & $\begin{array}{l}1.52 \\
1.26\end{array}$ & $\begin{array}{l}1.50 \\
1.27\end{array}$ & $\begin{array}{l}1.51 \\
1.25\end{array}$ & $\begin{array}{l}1.20 \\
1.24\end{array}$ & $\begin{array}{l}1.27 \\
1.23\end{array}$ \\
\hline 22 & $\begin{array}{l}\text { a. } \mathrm{CH} \\
\text { b. } \mathrm{CH}\end{array}$ & $\begin{array}{l}1.71 \\
1.41\end{array}$ & $\begin{array}{l}1.75 \\
1.52\end{array}$ & $\begin{array}{l}1.75 \\
1.50\end{array}$ & $\begin{array}{l}1.76 \\
1.25\end{array}$ & $\begin{array}{l}1.70 \\
1.53\end{array}$ & $\begin{array}{l}1.71 \\
1.53\end{array}$ \\
\hline 23 & $\mathrm{CH}_{3}$ & 1.11 & 1.11 & 1.11 & 1.11 & 1.11 & 1.11 \\
\hline 24 & $\mathrm{CH}_{3}$ & 1.10 & 1.10 & 1.10 & 1.10 & 1.10 & 1.10 \\
\hline 25 & $\mathrm{CH}_{3}$ & 1.60 & 1.15 & 1.15 & 1.14 & 1.16 & 1.15 \\
\hline 26 & $\mathrm{CH}_{3}$ & 1.16 & 1.16 & 1.16 & 1.17 & 1.16 & 1.18 \\
\hline 27 & $\mathrm{CH}_{3}$ & 1.28 & 1.27 & 1.26 & 1.25 & 1.28 & 1.30 \\
\hline 28 & $\mathrm{CO}$ & 1.06 & 1.05 & 1.04 & 1.05 & 1.05 & 1.04 \\
\hline 29 & $\mathrm{CH}_{3}$ & 1.10 & 1.05 & 1.05 & 1.06 & 1.05 & 1.06 \\
\hline 30 & $\mathrm{CH}_{3}$ & 7.90 & 8.70 & 7.66 & 8.80 & 8.05 & 8.08 \\
\hline 31 & $\mathrm{CONH}$ & 2.31 & 2.40 & 2.42 & 2.50 & 2.11 & 2.41 \\
\hline 32 & $\mathrm{NH}_{2}$ & 4.31 & 3.40 & 3.42 & 3.40 & 3.31 & 3.41 \\
\hline $1^{\prime}$ & $\mathrm{OCH}_{3}$ & - & - & 2.21 & 2.13 & - & - \\
\hline
\end{tabular}

tubes wereheated upto $50^{\circ} \mathrm{C}$. Solution of each test sample $(20 \mu \mathrm{g} / \mathrm{ml})$ was made in sterile DMSO. Ketoconazole in the same concentration, i.e., $20 \mu \mathrm{g} / \mathrm{ml}$ served as control drug. With the help of a micropipette, $100 \mu$ l solutions of test sample and control sample was added into different tubes of

TABLE 4B $\mid{ }^{1}$ HNMR spectral data of ligand and their metal (Tin) complexes.

Compound L $\delta(p p m) \mathrm{LSnPh}_{3} \mathrm{~L}_{2} \mathrm{SnPh}_{2} \mathrm{LSnBu}_{3} \mathrm{~L}_{2} \mathrm{SnBu}_{2} \mathrm{LSnMe}_{3} \mathrm{~L}_{2} \mathrm{SnMe}_{2}$

$\begin{array}{llllllll}\text { H \#. Atom } & \delta(\mathrm{ppm}) & \delta(\mathrm{ppm}) & \delta(\mathrm{ppm}) & \delta(\mathrm{ppm}) & \delta(\mathrm{ppm}) & \delta(\mathrm{ppm}) & \delta(\mathrm{ppm})\end{array}$

\begin{tabular}{|c|c|c|c|c|c|c|c|c|}
\hline 1 & $\begin{array}{l}\text { a. } \mathrm{CH} \\
\text { b. } \mathrm{CH}\end{array}$ & $\begin{array}{l}1.22 \\
1.18\end{array}$ & $\begin{array}{l}1.41 \\
1.20\end{array}$ & $\begin{array}{l}1.43 \\
1.20\end{array}$ & $\begin{array}{l}1.41 \\
1.20\end{array}$ & $\begin{array}{l}1.43 \\
1.28\end{array}$ & $\begin{array}{l}1.46 \\
1.23\end{array}$ & $\begin{array}{l}1.43 \\
1.20\end{array}$ \\
\hline 2 & $\begin{array}{l}\text { a.CH } \\
\text { b.CH }\end{array}$ & $\begin{array}{l}1.21 \\
1.18\end{array}$ & $\begin{array}{l}1.71 \\
1.47\end{array}$ & $\begin{array}{l}1.71 \\
1.48\end{array}$ & $\begin{array}{l}1.70 \\
1.47\end{array}$ & $\begin{array}{l}1.72 \\
1.47\end{array}$ & $\begin{array}{l}1.78 \\
1.45\end{array}$ & $\begin{array}{l}1.72 \\
1.38\end{array}$ \\
\hline 3 & $\mathrm{CH}$ & 2.99 & 3.00 & 3.17 & 3.15 & 3.09 & 3.30 & 3.10 \\
\hline $3^{\prime}$ & $\mathrm{OH}$ & 3.15 & 2.50 & 2.29 & 2.50 & 2.51 & 2.30 & 2.98 \\
\hline 4 & - & - & - & - & - & - & - & - \\
\hline 5 & $\mathrm{CH}$ & 1.45 & 1.38 & 1.30 & 1.31 & 1.33 & 1.36 & 1.35 \\
\hline 6 & $\begin{array}{l}\text { a. } \mathrm{CH} \\
\text { b. } \mathrm{CH}\end{array}$ & $\begin{array}{l}1.09 \\
1.01\end{array}$ & $\begin{array}{l}1.57 \\
1.20\end{array}$ & $\begin{array}{l}1.06 \\
1.20\end{array}$ & $\begin{array}{l}1.50 \\
1.20\end{array}$ & $\begin{array}{l}1.51 \\
1.21\end{array}$ & $\begin{array}{l}1.57 \\
1.21\end{array}$ & $\begin{array}{l}1.57 \\
1.21\end{array}$ \\
\hline 7 & $\begin{array}{l}\text { a. } \mathrm{CH} \\
\text { b.CH }\end{array}$ & $\begin{array}{l}1.22 \\
1.18\end{array}$ & $\begin{array}{l}1.47 \\
1.20\end{array}$ & $\begin{array}{l}1.48 \\
1.20\end{array}$ & $\begin{array}{l}1.40 \\
1.24\end{array}$ & $\begin{array}{l}1.41 \\
1.25\end{array}$ & $\begin{array}{l}1.40 \\
1.23\end{array}$ & $\begin{array}{l}1.41 \\
1.27\end{array}$ \\
\hline 8 & - & - & - & - & - & - & - & - \\
\hline 9 & $\mathrm{CH}$ & 1.43 & 1.45 & 1.43 & 1.42 & 1.43 & 1.46 & 1.43 \\
\hline 10 & - & - & - & - & - & - & - & - \\
\hline 11 & $\begin{array}{l}\text { a.CH } \\
\text { b. } \mathrm{CH}\end{array}$ & $\begin{array}{l}1.24 \\
1.22\end{array}$ & $\begin{array}{l}1.26 \\
1.21\end{array}$ & $\begin{array}{r}2.05 \\
1.70\end{array}$ & $\begin{array}{l}2.04 \\
1.71\end{array}$ & $\begin{array}{l}2.05 \\
1.70\end{array}$ & $\begin{array}{l}2.06 \\
1.78\end{array}$ & $\begin{array}{r}2.20 \\
1.70\end{array}$ \\
\hline 12 & $\mathrm{CH}$ & 5.01 & 5.21 & 4.66 & 4.67 & 5.13 & 5.13 & 4.64 \\
\hline 13 & $\mathrm{C}$ & - & - & - & - & - & - & - \\
\hline 14 & C & - & - & - & - & - & - & - \\
\hline 15 & $\begin{array}{l}\text { a. } \mathrm{CH} \\
\text { b. } \mathrm{CH}\end{array}$ & $\begin{array}{l}1.22 \\
1.19\end{array}$ & $\begin{array}{l}1.21 \\
1.18\end{array}$ & $\begin{array}{l}1.43 \\
1.17\end{array}$ & $\begin{array}{l}1.37 \\
1.12\end{array}$ & $\begin{array}{l}1.31 \\
1.10\end{array}$ & $\begin{array}{l}1.31 \\
1.09\end{array}$ & $\begin{array}{l}1.32 \\
1.08\end{array}$ \\
\hline 16 & $\begin{array}{l}\text { a. } \mathrm{CH} \\
\text { b. } \mathrm{CH}\end{array}$ & $\begin{array}{l}1.22 \\
1.18\end{array}$ & $\begin{array}{l}1.22 \\
1.18\end{array}$ & $\begin{array}{l}1.60 \\
1.32\end{array}$ & $\begin{array}{l}1.62 \\
1.31\end{array}$ & $\begin{array}{l}1.63 \\
1.30\end{array}$ & $\begin{array}{l}1.55 \\
1.31\end{array}$ & $\begin{array}{l}1.54 \\
1.13\end{array}$ \\
\hline 17 & - & - & - & - & - & - & - & - \\
\hline 18 & $\mathrm{CH}$ & 2.65 & 2.76 & 2.31 & 2.50 & 2.51 & 2.76 & 2.77 \\
\hline 19 & $\begin{array}{l}\text { a. } \mathrm{CH} \\
\text { b. } \mathrm{CH}\end{array}$ & $\begin{array}{l}1.52 \\
1.35\end{array}$ & $\begin{array}{l}1.62 \\
1.32\end{array}$ & $\begin{array}{l}1.62 \\
1.61\end{array}$ & $\begin{array}{l}1.60 \\
1.62\end{array}$ & $\begin{array}{l}1.61 \\
1.61\end{array}$ & $\begin{array}{l}1.55 \\
1.60\end{array}$ & $\begin{array}{l}1.54 \\
1.67\end{array}$ \\
\hline 20 & - & - & - & 1.50 & 1.51 & 1.52 & 1.50 & 1.51 \\
\hline 21 & $\begin{array}{l}\text { a.CH } \\
\text { b. } \mathrm{CH}\end{array}$ & $\begin{array}{l}1.35 \\
1.22\end{array}$ & $\begin{array}{l}1.29 \\
1.20\end{array}$ & $\begin{array}{l}1.21 \\
1.70\end{array}$ & $\begin{array}{l}1.20 \\
1.71\end{array}$ & $\begin{array}{l}1.21 \\
1.71\end{array}$ & $\begin{array}{l}1.21 \\
1.71\end{array}$ & $\begin{array}{l}1.21 \\
1.72\end{array}$ \\
\hline 22 & $\begin{array}{l}\text { a. } \mathrm{CH} \\
\text { a.CH }\end{array}$ & $\begin{array}{l}1.35 \\
1.22\end{array}$ & $\begin{array}{l}1.29 \\
1.21\end{array}$ & $\begin{array}{l}1.51 \\
1.11\end{array}$ & $\begin{array}{l}1.50 \\
1.11\end{array}$ & $\begin{array}{l}1.52 \\
1.11\end{array}$ & $\begin{array}{l}1.51 \\
1.11\end{array}$ & $\begin{array}{l}1.52 \\
1.11\end{array}$ \\
\hline 23 & $\mathrm{CH}_{3}$ & 0.72 & 0.70 & 1.10 & 1.10 & 1.10 & 1.10 & 1.10 \\
\hline 24 & $\mathrm{CH}_{3}$ & 0.71 & 0.73 & 1.16 & 1.15 & 1.14 & 1.15 & 1.12 \\
\hline 25 & $\mathrm{CH}_{3}$ & 0.65 & 0.65 & 1.17 & 1.16 & 1.17 & 1.16 & 1.17 \\
\hline 26 & $\mathrm{CH}_{3}$ & 0.85 & 0.86 & 1.27 & 1.28 & 1.29 & 1.27 & 1.28 \\
\hline 27 & $\mathrm{CH}_{3}$ & 1.07 & 1.06 & - & - & - & - & - \\
\hline 28 & $\mathrm{CO}$ & - & - & 1.05 & 1.06 & 1.05 & 1.07 & 1.08 \\
\hline 29 & $\mathrm{CH}_{3}$ & 0.65 & 0.65 & 0.73 & 0.73 & 0.66 & 0.77 & 0.74 \\
\hline 30 & $\mathrm{CH}_{3}$ & 0.71 & 0.72 & 7.40 & 7.25 & 7.42 & 7.26 & 7.27 \\
\hline 31 & $\mathrm{CONH}$ & 8.01 & 7.38 & 3.7 & 2.88 & 2.80 & 2.31 & 2.53 \\
\hline 32 & $\mathrm{NH}_{2}$ & 4.21 & 3.6 & 6.88 & $\begin{array}{c}1.3 \\
1.32,1.3 \\
0.93\end{array}$ & $\begin{array}{c}1.3, \\
1.32,1.3, \\
0.93\end{array}$ & 0.89 & 0.87 \\
\hline $1^{\prime}$ & Ph. H & - & 7.7 & - & - & - & - & - \\
\hline $2^{\prime}$ & $\begin{array}{l}\text { Butyl and } \\
\text { Methyl } \\
\text { Hydrogen }\end{array}$ & - & - & - & $\begin{array}{l}1.2 \\
1.22 \\
1.23 \\
0.83\end{array}$ & $\begin{array}{l}1.2 \\
1.22 \\
1.23 \\
0.83\end{array}$ & 0.67 & 0.6 \\
\hline
\end{tabular}


non-solidified agar media. The tubes were allowed to solidify at room temperature. Each of the tube was inoculated with inoculums of $4 \mathrm{~mm}$ diameter piece which was obtained from 7 days old fungal strains' culture. Then all tubes were incubated at optimum temperature, i.e., $28-30^{\circ} \mathrm{C}$ for $7-10$ days. The controlled humidity (40-50\%) was achieved with the help of an open pan of water in the incubator. During incubation, the cultures were observed at least twice a week. After 710 days of incubation, minimum inhibitory concentration (MIC in $\mu \mathrm{g} / \mathrm{mL}$ )MIC was calculated as the lowest concentration of the extract inhibiting the growth of bacterial strainto obtain a final concentration ranging from 256 to $0.16 \mu \mathrm{g} / \mathrm{ml}$. The assay was performed as triplicate analysis (Irobi et al., 1996).

\section{Determination of Antioxidant Activity}

The DPPH assay (DPPH free radicals) was used to find out the anti-radical properties of the samples (Ahmad et al., 2015). DPPH solution was prepared by adding $3.2 \mathrm{mg}$ of it into $100 \mathrm{ml}$ methanol (82\%). Into a glass vial, $2,800 \mu \mathrm{l}$ of DPPH solution was added followed by $200 \mu \mathrm{l}$ of test sample. The serial dilutions were prepared in concentration of $1,000,500,250,125$, and $62.5 \mu \mathrm{g} / \mathrm{ml}$.

The mixture was stirred and allowed to stay at room temperature in dark for $1 \mathrm{~h}$. UV-Visible Spectrophotometer (Shimadzu 1,800) was used for recording of absorbance. A mixture of $82 \%$ methanol $(2,800 \mu \mathrm{l})$ and $200 \mu \mathrm{l}$ methanol was used as blank. Whilemethanol 200 and 2,800 $\mu$ l of DPPH solution was used as a control. The test was repeated three (3) times. The
$\%$ inhibition was find out by using the given formula.

$$
\text { Scavenging effect }(\%)=\frac{A_{c}-A_{s}}{A_{c}} \times 100
$$

Where $A_{c}$ is the control absorbance and $A_{s}$ is the test sample absorbance.

\section{Docking Studies}

Docking studies were carried out using Autodock 4.2. Three dimensional (3D) crystal structures of the enzymes retrieved from Protein Data Bank (PDB). For the study of antioxidant activity, molecular docking studies were carried out human peroxiredoxins ( $\mathrm{PrxV}, \mathrm{PDB}$ code $3 \mathrm{MNG}$ ) with a competitive antioxidant inhibitor 1,2-dithiane-4,5-diol (DTT) was retrieved from Protein Data Bank (PDB). While, for study of the binding orientation for the antibacterial study, penicillin binding protein (PBP) with PDB code 2EX9 with penicillin $\mathrm{V}$ as co-crystallized ligand was retrieved.

The structures of the metal complexes were drawn using Marvin sketch 16.5.2. The 3D structures of these complexes were optimized using prepare ligand module in Autodock 4.2. The parameter file of AutoDock was modified to incorporate metalVan der Waals and other needed parameters which were obtained from the AutoDock website. For enzyme downloaded from PDB, solvation parameters and Kollman charges for all the atoms were assigned. AutoDock Tools (ADT) were used to create PDBQT file for both ligand and enzyme. A grid parameter file was generated using ADT. A cubic grid box of $60 \AA$ (x, y, z) (for
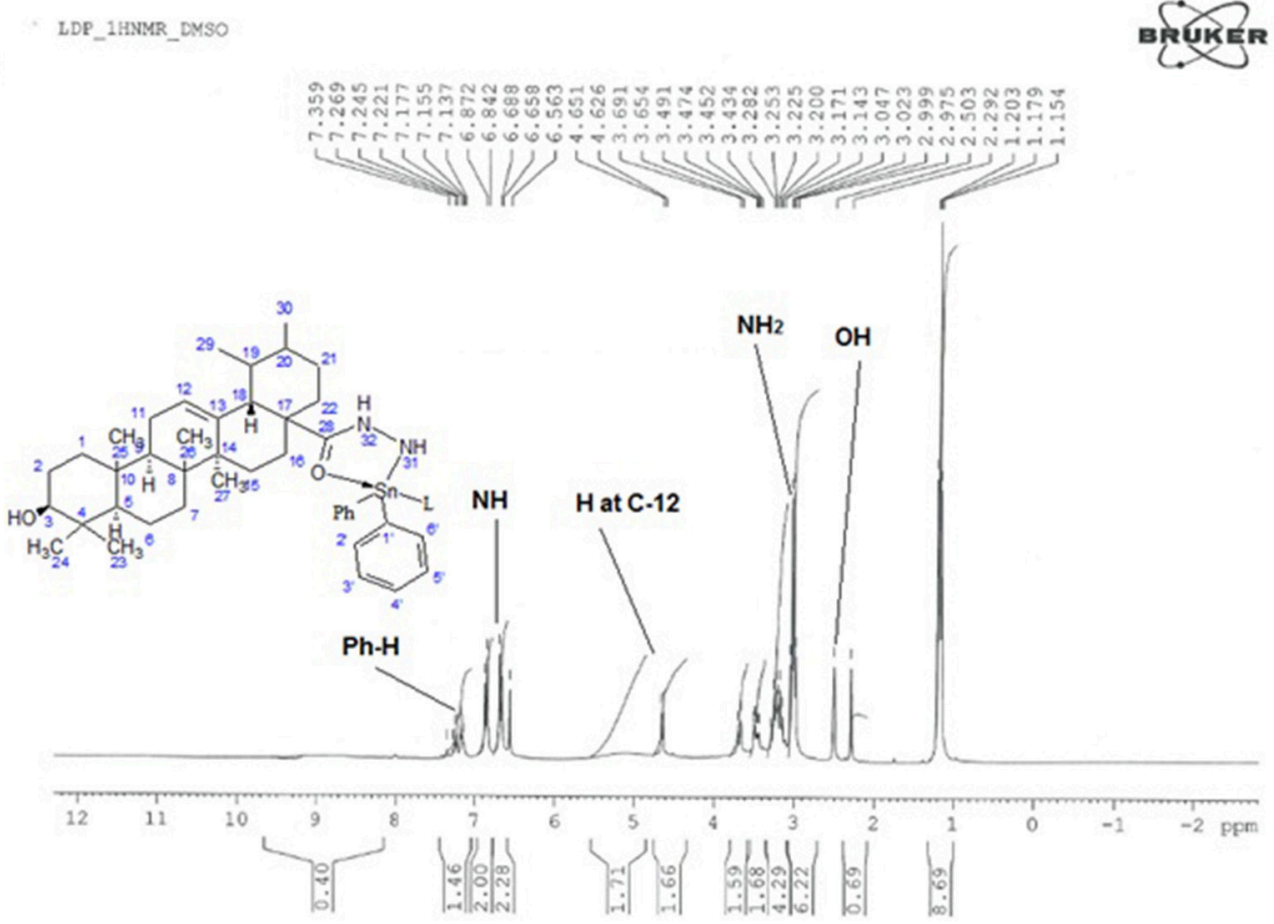

FIGURE 5 | ${ }^{1} \mathrm{H}$ NMR Spectrum of Diphenyltin complex. 
$3 \mathrm{MNG}$ ) and a cubic grid box of $45 \AA$ (x, y, z) (for 2EX9) with a spacing of $0.375 \AA$ was created.

The reliability of docking program was validated by using re-docking method. In both cases the co-crystallized ligands were re-docked into the active site of the downloaded enzymes. Root Mean Square (RMSD) was then calculated between the cocrystallized and re-docked poses. In both case, the RMSD value are within the threshold limit. In all cases, RMSD value of $<2.0$ $\AA$ is considered as accurate in predicting binding orientation of ligand. The binding poses were studied using discovery studio visualizer.

\section{RESULTS AND DISCUSSIONS}

\section{Physical Data and Solubility}

The synthesis of the ligand is shown in Figure $\mathbf{1}$ while its metal complexation is provided in Figures 2, 3. The physical data and solubility of ligand and metals complexes have been presented

TABLE 5A $\mid{ }^{13} \mathrm{CNMR}$ spectral data of ligand and their metal $(\mathrm{Cu} / \mathrm{Sb} / \mathrm{Zn} / \mathrm{Fe})$ complexes.

Compound UA Ligand (L) $\mathrm{LFeCl}_{2} \mathrm{LCu}(\mathrm{Ac})_{2} \mathrm{LZn}(\mathrm{Ac})_{2} \mathrm{LSbCl}_{2} \mathrm{LSbBr}_{2}$

C No. Atom $\delta(\mathrm{ppm}) \quad \delta(\mathrm{ppm}) \quad \delta(\mathrm{ppm}) \quad \delta(\mathrm{ppm}) \quad \delta(\mathrm{ppm}) \quad \delta(\mathrm{ppm}) \delta(\mathrm{ppm})$

\begin{tabular}{|c|c|c|c|c|c|c|c|c|}
\hline 1 & $\mathrm{CH}_{2}$ & 38 & 39.97 & 39.5 & 39.5 & 39.7 & 39.90 & 39.03 \\
\hline 2 & $\mathrm{CH}_{2}$ & 27.7 & 27.24 & 27.1 & 27.45 & 27.4 & 27.6 & 27.82 \\
\hline 3 & $\mathrm{CH}$ & 78.5 & 80.3 & 78.7 & 80.64 & 80.6 & 78.61 & 79.62 \\
\hline 4 & C & 39.5 & 40.94 & 40.5 & 41.42 & 40.4 & 40.37 & 41.4 \\
\hline 5 & $\mathrm{CH}$ & 55.9 & 54.33 & 54 & 53 & 54.4 & 54.26 & 55.8 \\
\hline 6 & $\mathrm{CH}_{2}$ & 19 & 20.9 & 20 & 20.8 & 20.9 & 20.8 & 20.9 \\
\hline 7 & $\mathrm{CH}_{2}$ & 34.3 & 35.35 & 35.3 & 35.5 & 35.1 & 35.07 & 36.09 \\
\hline 8 & C & 39.8 & 39.70 & 40 & 40.7 & 40.9 & 40.9 & 40.92 \\
\hline 9 & $\mathrm{CH}$ & 48.5 & 47.81 & 48 & 48.6 & 47.7 & 48.84 & 49.55 \\
\hline 10 & C & 37.5 & 37.1 & 38.5 & 38.1 & 38.08 & 38.03 & 39.04 \\
\hline 11 & $\mathrm{CH}_{2}$ & 25.7 & 25.7 & 25 & 25.07 & 25 & 25.82 & 25.83 \\
\hline 12 & $\mathrm{CH}$ & 121.6 & 118.1 & 118.9 & 118.97 & 118.9 & 118.97 & 118.98 \\
\hline 13 & C & 145.8 & 150.7 & 150.2 & 150.2 & 150.2 .4 & 150.2 & 152.26 \\
\hline 14 & C & 42.5 & 42.5 & 42.5 & 42.72 & 42.5 & 42.72 & 41.93 \\
\hline 15 & $\mathrm{CH}_{2}$ & 28.5 & 29.4 & 29.6 & 29.65 & 29.6 & 29.95 & 29.66 \\
\hline 16 & $\mathrm{CH}_{2}$ & 23.7 & 28.8 & 28.6 & 28.09 & 28.1 & 28.06 & 27.07 \\
\hline 17 & $\mathrm{C}$ & 45.7 & 44.7 & 45 & 45.08 & 44.5 & 45.71 & 45.37 \\
\hline 18 & $\mathrm{CH}$ & 42 & 54.0 & 54 & 54.7 & 54.6 & 55.5 & 54.6 \\
\hline 19 & $\mathrm{CH}$ & 46.0 & 39.5 & 39 & 40.3 & 39.5 & 40.56 & 41.57 \\
\hline 20 & $\mathrm{CH}$ & 30.5 & 38.1 & 38.8 & 39.6 & 39.9 & 39.83 & 38.84 \\
\hline 21 & $\mathrm{CH}_{2}$ & 34.5 & 29.31 & 30 & 29.4 & 30.4 & 29.3 & 28.93 \\
\hline 22 & $\mathrm{CH}_{2}$ & 33.5 & 35.7 & 33.7 & 34.5 & 33.7 & 35.33 & 35.34 \\
\hline 23 & $\mathrm{CH}_{3}$ & 28.9 & 18.8 & 19.5 & 18.9 & 18.9 & 18.9 & 18.92 \\
\hline 24 & $\mathrm{CH}_{3}$ & 17.6 & 19.5 & 19.5 & 19.0 & 19.0 & 19.0 & 19.47 \\
\hline 25 & $\mathrm{CH}_{3}$ & 20.6 & 22.66 & 21.7 & 20.5 & 21.7 & 20.5 & 21.56 \\
\hline 26 & $\mathrm{CH}_{3}$ & 17.5 & 20.6 & 18.9 & 19.5 & 19.0 & 19.2 & 18.9 \\
\hline 27 & $\mathrm{CH}_{3}$ & 26.5 & 25 & 21 & 21 & 21 & 21.0 & 26.07 \\
\hline 28 & C & 180.2 & 180 & 181 & 180 & 180 & 178.0 & 179.08 \\
\hline 29 & $\mathrm{CH}_{3}$ & 33.5 & 16.7 & 16.7 & 17.7 & 17.5 & 17.00 & 18.0 \\
\hline 30 & $\mathrm{CH}_{3}$ & 21.8 & 18.81 & 19 & 18.6 & 19.5 & 19.02 & 20.0 \\
\hline 31 & $\mathrm{C}$ & & & & 175 & & & \\
\hline 32 & $\mathrm{CH}_{3}$ & & & & 23.0 & & & \\
\hline
\end{tabular}

in the Tables 1, 2 respectively. The abbreviations of various complexes have also been mentioned in these tables.

\section{IR Spectral Analysis and IR Spectrum of Dipheyltin Complex}

The Figure 4 and Table 3 shows the most important bands of FT-IR spectra recorded for all the ligands and complexes. The attachment of metals can be found out by the absence of primary stretch in the metal complexes from the ligand. The metal complex can be confirmed from the appearance of new bands in FT-IR, which are not present in the ligand's spectrum. The band of NH has been shifted to the lower band area i.e., about 30

TABLE 5B | ${ }^{13}$ CNMR spectral data of ligand and their metal (Tin) complexes.

Compound L $\quad \mathrm{Ph}_{3} \mathrm{SnL} \mathrm{Ph}_{2} \mathrm{SnL}_{2} \mathrm{Bu}_{3} \mathrm{SnL} \mathrm{Bu}_{2} \mathrm{SnL}_{2} \mathrm{Me}_{3} \mathrm{SnL} \mathrm{Me}_{2} \mathrm{SnL}_{2}$

\begin{tabular}{|c|c|c|c|c|c|c|c|c|}
\hline C No & Atom & $\delta(\mathrm{ppm})$ & $\delta(\mathrm{ppm})$ & $\delta(\mathrm{ppm})$ & $\delta(\mathrm{ppm})$ & $\delta(\mathrm{ppm})$ & $\delta(\mathrm{ppm})$ & $\delta(\mathrm{ppm})$ \\
\hline 1 & $\mathrm{CH}_{2}$ & 33.97 & 34.30 & 33.0 & 33.08 & 33.19 & 33.01 & 33.18 \\
\hline 2 & $\mathrm{CH}_{2}$ & 27.24 & 27.24 & 27.25 & 27.26 & 27.05 & 27.04 & 27.69 \\
\hline 3 & $\mathrm{CH}$ & 80.3 & 77.09 & 77.39 & 77.35 & 77.09 & 80.33 & 79.73 \\
\hline 4 & C & 40.94 & 41.91 & 40.93 & 41.95 & 41.56 & 41.03 & 39.11 \\
\hline 5 & $\mathrm{CH}$ & 54.33 & 45.93 & 45.33 & 54.35 & 53.37 & 53.32 & 53.34 \\
\hline 6 & $\mathrm{CH}_{2}$ & 20.9 & 17.91 & 21.92 & 20.01 & 20.91 & 20.01 & 20.4 \\
\hline 7 & $\mathrm{CH}_{2}$ & 35.35 & 35.37 & 39.38 & 33.04 & 35.34 & 35.32 & 35.30 \\
\hline 8 & C & 39.70 & 39.92 & 39.93 & 39.94 & 39.93 & 39.07 & 39.93 \\
\hline 9 & $\mathrm{CH}$ & 47.81 & 47.81 & 47.82 & 47.85 & 47.85 & 47.40 & 47.65 \\
\hline 10 & C & 37.1 & 39.8 & 39.82 & 37.98 & 37.82 & 37.90 & 37.96 \\
\hline 11 & $\mathrm{CH}_{2}$ & 25.7 & 25.78 & 21.79 & 25.77 & 25.78 & 25.74 & 25.67 \\
\hline 12 & $\mathrm{CH}$ & 118.1 & 115.64 & 118.05 & 118.65 & 118.06 & 118.91 & 118.9 \\
\hline 13 & C & 152.7 & 144.2 & 137.1 & 150.12 & 144.28 & 144.20 & 152.12 \\
\hline 14 & C & 42.5 & 45.50 & 40.70 & 42.5 & 42.70 & 42.67 & 42.95 \\
\hline 15 & $\mathrm{CH}_{2}$ & 29.4 & 29.30 & 29.35 & 29.3 & 29.46 & 29.63 & 29.43 \\
\hline 16 & $\mathrm{CH}_{2}$ & 28.8 & 28.90 & 29.91 & 28.93 & 28.98 & 28.96 & 28.6 \\
\hline 17 & C & 44.7 & 45.03 & 45.04 & 44.04 & 44.07 & 44.02 & 44.01 \\
\hline 18 & $\mathrm{CH}$ & 54.0 & 61.00 & 61.19 & 54.2 & 52.21 & 54.17 & 54.95 \\
\hline 19 & $\mathrm{CH}$ & 39.5 & 39.03 & 39.04 & 39.53 & 39.45 & 39.42 & 39.79 \\
\hline 20 & $\mathrm{CH}$ & 38.1 & 39.80 & 39.81 & 39.91 & 38.51 & 38.49 & 38.88 \\
\hline 21 & $\mathrm{CH}_{2}$ & 29.31 & 29.40 & 29.45 & 29.5 & 29.54 & 29.51 & 29.54 \\
\hline 22 & $\mathrm{CH}_{2}$ & 35.7 & 33.71 & 33.14 & 33.75 & 33.14 & 33.71 & 33.70 \\
\hline 23 & $\mathrm{CH}_{3}$ & 18.8 & 18.95 & 18.51 & 18.92 & 19.01 & 19.50 & 18.69 \\
\hline 24 & $\mathrm{CH}_{3}$ & 19.5 & 19.49 & 19.55 & 19.35 & 19.60 & 19.50 & 19.57 \\
\hline 25 & $\mathrm{CH}_{3}$ & 22.66 & 15.60 & 21.50 & 21.53 & 25.22 & 21.70 & 21.63 \\
\hline 26 & $\mathrm{CH}_{3}$ & 20.6 & 18.31 & 18.34 & 18.4 & 18.45 & 18.42 & 18.44 \\
\hline 27 & $\mathrm{CH}_{3}$ & 25 & 21.00 & 21.02 & 21.0 & 25.2 & 21.19 & 21.12 \\
\hline 28 & C & 180 & 152 & 152.71 & 178.3 & 152 & 180.9 & 179 \\
\hline 29 & $\mathrm{CH}_{3}$ & 16.7 & 16.72 & 16.74 & 16.95 & 16.89 & 16.74 & 16.30 \\
\hline 30 & $\mathrm{CH}_{3}$ & 18.81 & 18.91 & 18.80 & 18.9 & 18.4 & 18.90 & 18.86 \\
\hline 1 & C & & 137.67 & 137.67 & 8.3 & 11.10 & & -1.4 \\
\hline 2 & $\mathrm{CH}$ & & 128.30 & 128.30 & 26.3 & 23.40 & & -1.4 \\
\hline 3 & $\mathrm{CH}$ & & 129.3 & 129.36 & 13.8 & 13.80 & & \\
\hline 4 & $\mathrm{CH}$ & & 128.2 & 128.22 & & & & \\
\hline $5^{\prime}$ & $\mathrm{CH}$ & & 128.2 & 128.22 & & & & \\
\hline 6 & $\mathrm{CH}$ & & 136.8 & 136.8 & & & & \\
\hline
\end{tabular}


$\mathrm{cm}^{-1}$ due to coordination. The NH groups showed no significant shift in its fundamental shift. This confirms that negligible interaction is there between metal complexes and $\mathrm{N}=\mathrm{O}$ group. The IR investigation reveals that designed molecular structures of metal complexes go parallel with the observations from the experiments. The $\mathrm{NH}_{2}, \mathrm{C}=\mathrm{O}, \mathrm{C}-\mathrm{O}, \mathrm{NH}$ and $\mathrm{C}=\mathrm{C}$ peaks can be seen in the ligand's spectrum. The $\mathrm{NH}_{2}, \mathrm{NH}, \mathrm{C}-\mathrm{O}$ and $\mathrm{C}=\mathrm{C}$ stretches have been recorded in 3,360, 3,200, 1,215, and 1,650 $\mathrm{cm}^{-1}$ respectively. The new complex formation can also be confirmed from the new peaks in zinc complex spectrum. The M$\mathrm{O}$ and $\mathrm{M}-\mathrm{N}$ can be observed in 400 and $594 \mathrm{~cm}^{-1}$ respectively. The $\mathrm{NH} 2, \mathrm{C}=\mathrm{C}$ aromatic, $\mathrm{C}-\mathrm{C}, \mathrm{C}=\mathrm{O}$ and $\mathrm{NH}$ peaks have also been shown in the spectrum. The peaks of $\mathrm{M}-\mathrm{O}$ and $\mathrm{M}-\mathrm{N}$ in the diphenyl tin complex spectrum can be seen at 400 and above 450 $\mathrm{cm}^{-1}$ respectively. The $\mathrm{C}-\mathrm{O}, \mathrm{C}=\mathrm{C}, \mathrm{C}=\mathrm{O}, \mathrm{NH} 2$ and $\mathrm{NH}$ peaks appear at 1,211,1,650 to $1,700,1,508,3,300$, and $3,151 \mathrm{~cm}^{-1}$ respectively. So the molecular structure of diphenyl tin complex was confirmed from the data obtained.

\section{${ }^{1}$ H NMR Spectroscopy}

The proposed molecular structure of compounds was confirmed from the peaks in ${ }^{1}$ HNMR spectrum of metal complexes and ligand. The ${ }^{1} \mathrm{H}$ NMR data of compounds with various complexes have been presented in Tables 4A,B. The hydrogen of hydroxyl group, $\mathrm{NH} 2$ group and phenyl group have been shown at 2.4, 3.4, and 7.2 chemical shifts respectively as shown in Figure 5. So this analysis confirms the structure.

\section{${ }^{13} \mathrm{C}$ NMR Spectral Analysis}

The ${ }^{13} \mathrm{C}$-NMR spectroscopy of metal complexes and ligand were carried out at $300 \mathrm{MHz}$ on Bruker NMR spectrometer.
The solvent used for dissolution were DMSO and chloroform. The data obtained were summarized in Tables $\mathbf{5 A}, \mathbf{B}$. The amide bond with UAconfirms the formation of ligand, which appears at 179 chemical shift. The peaks of metal complexes were detected between 9 and 10. The presence of $\mathrm{OH}$ in all metal complexes has been confirmed by $2-3$ peaks. The C-28 and

TABLE 6 | MIC values of synthesized metal complexes.

\begin{tabular}{lcccc}
\hline Compound No. & \multicolumn{4}{c}{ Antibacterial activity (MIC in $\boldsymbol{\mu} \mathbf{g} / \mathbf{m l})^{\mathbf{a}}$} \\
\cline { 2 - 5 } & Shigella & S. typhi & S. aureus & S. pneumonia \\
\hline UL & $>256$ & $>256$ & $>256$ & $>256$ \\
LCu & $>256$ & $>256$ & 128 & $>256$ \\
LZn & 128 & $>256$ & $>256$ & 128 \\
LFe & 32 & 64 & 32 & 32 \\
LSC & 32 & 32 & 64 & 32 \\
LSB & 32 & 32 & 32 & 32 \\
LDM & 32 & 32 & 16 & 16 \\
LTM & 32 & 32 & 32 & 32 \\
LDB & 16 & 16 & 16 & 16 \\
LTB & 16 & 16 & $\mathbf{8}$ & $\mathbf{8}$ \\
LDP & $\mathbf{8}$ & $\mathbf{8}$ & $\mathbf{8}$ & $\mathbf{8}$ \\
LTP & $\mathbf{8}^{\mathbf{b}}$ & $\mathbf{8}$ & $\mathbf{8}$ & $\mathbf{4}$ \\
Ciprofloxacin & 8 & $N P C$ & 8 & NP \\
Penicillin V & 0.01 & 0.01 & 0.05 & 0.05 \\
\hline
\end{tabular}

aThe reported MIC values in $\mu \mathrm{g} / \mathrm{ml}$ are an average of at least three individual measurements.

${ }^{b}$ The values shown in bold are the better/equal inhibitors than the standard drugs used. ${ }^{c} N P$, Not performed.

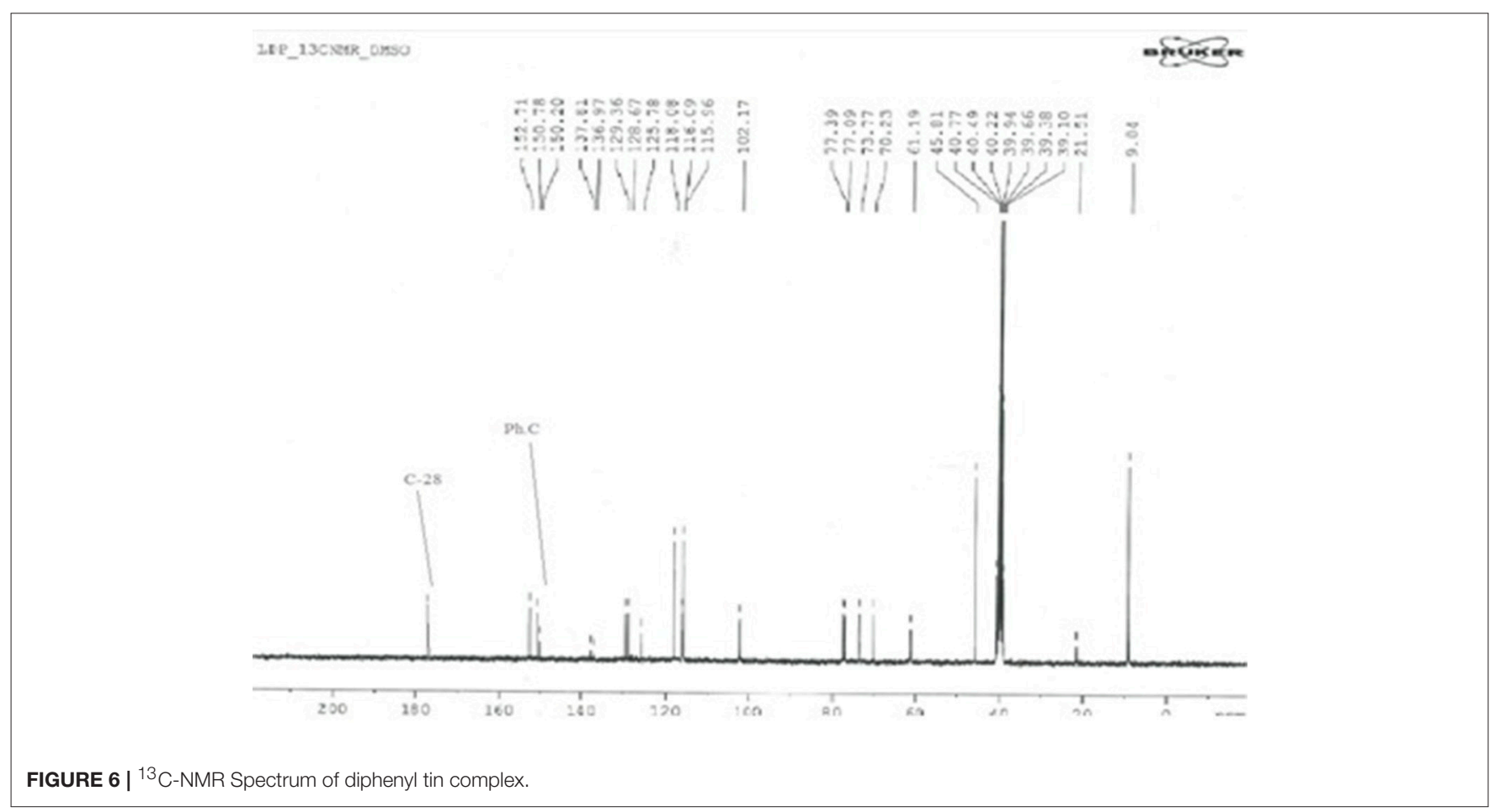


phenyl carbon in diphenyl tin complex have been presented by the peaks at 179 and 154 chemical shifts respectively as shown in Figure 6.

\section{Biological Assays}

\section{Antibacterial Activity}

For the screening of antibacterial activity, Agar Well Diffusion assay was carried out. The Tetracycline was used as positive control in the study. The results of antibacterial assay have been presented in Table 6. Synthesized ligand (UL) has shown lowest activity with MIC value of $128 \mu \mathrm{g} / \mathrm{mL}$ against all studied strains. The antibacterial activity of metal complexes was recorded higher as compared to the ligands. Triphenyl tin complex (LTP) emerged as potent antibacterial agent with MIC value of $8 \mu \mathrm{g} / \mathrm{mL}$ each against Shigellaspp, S. typhi, and S. aureus. While, the MIC value against Streptococcus pneumoniae is $4 \mu \mathrm{g} / \mathrm{mL}$. Similarly, tributyl tin complex (LTB) also showed excellent activity against Grampositive species $S$. aureus and $S$. pneumoniae $(8 \mu \mathrm{g} / \mathrm{mL}$ each, Table 6). The MIC value of LTB against Gram-negative strain
(Shigellaspp and S. typhi) is $16 \mu \mathrm{g} / \mathrm{mL}$. Diphenyl tin complex (LDP) showed MIC value of $8 \mu \mathrm{g} / \mathrm{mL}$ for each strain. Dibutyl tin (LDB) and dimethyl tin (LDM) complexes also showed good activities. LDB with MIC value of $8 \mu \mathrm{g} / \mathrm{mL}$ exhibited excellent activity against $S$. pneumoniae. However, copper and zinc complexes were not able to show good activities. Two antimony complexes have shown the activity to some extent. Bromo antimony complex exhibited MIC value of $32 \mu \mathrm{g} / \mathrm{mL}$ against each strain.

\section{Antioxidant Activity}

The antioxidant activity shown by the metal complexes and ligands were recorded as significant. In this assay ascorbic acid was employed as positive control. The radicals scavenging activity of metal complexes was recorded as higher as compared to the ligands. The highest antioxidant activity has been shown by the triphenyl tin complex as shown in the Figure 7. Similarly, the least antioxidant activity has been revealed by the antimony

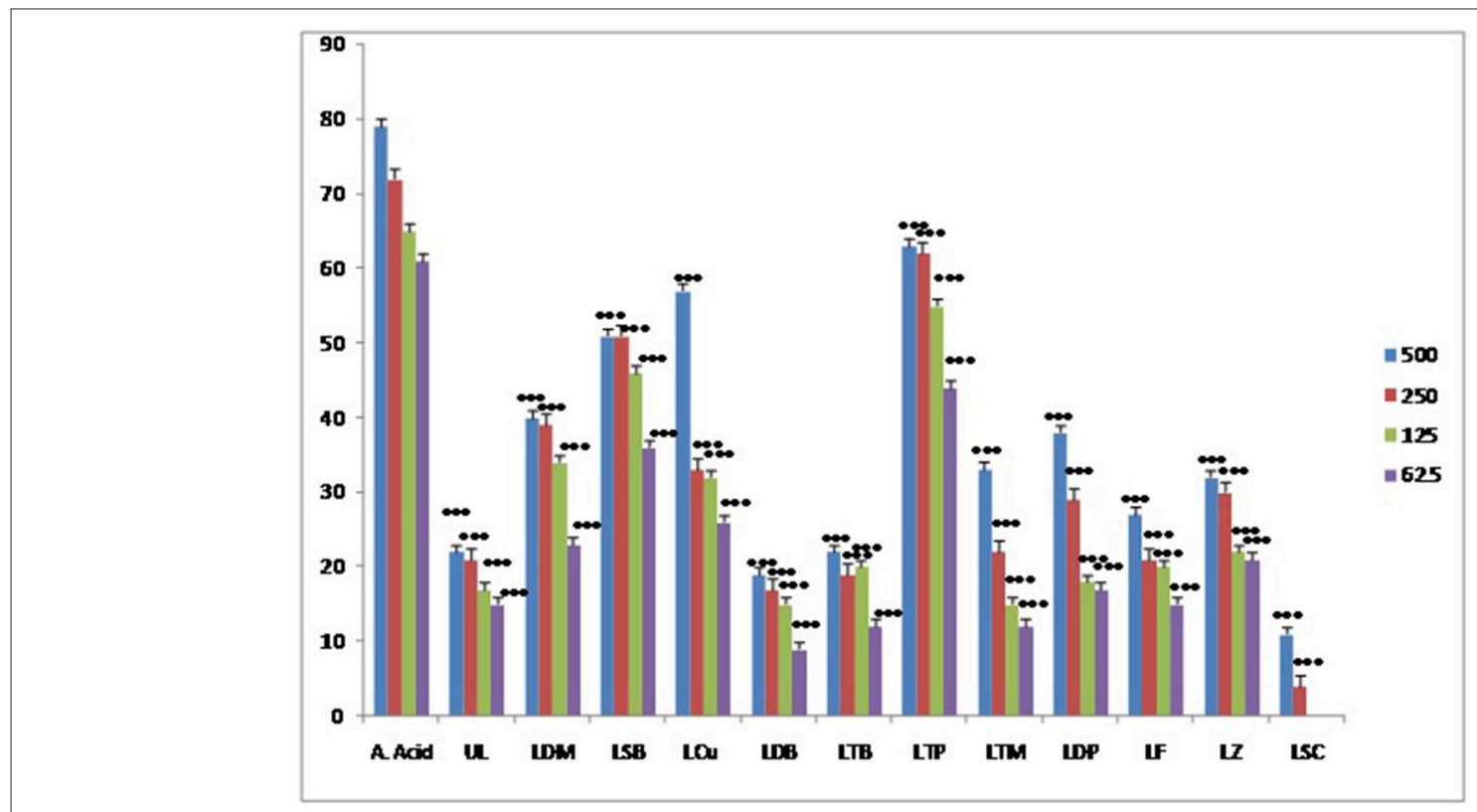

FIGURE 7 | Results of antioxidant activity of various samples.
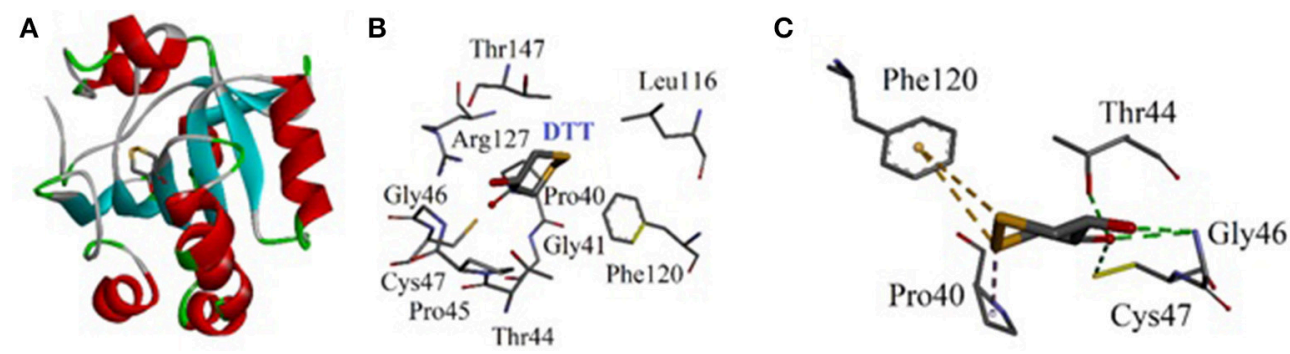

FIGURE 8 | (A) Computer generated structure ofhuman PrxV (PDB code 3MNG) with a competitive antioxidant inhibitor 1,2-dithiane-4,5-diol (DTT) (ribbon form); (B) Active site of 3MNG; (C) Interactions of DTT with important residues. 
trichloride complex as the activity of this complex come to the baseline i.e., zero at $125 \mu \mathrm{g} / \mathrm{ml}$.

Various compounds, either natural or synthetic are inevitable for the life of human on this planet. The compounds which are bestowed by the nature are not in the final shape (Leplege and Hunt, 1997). These are given in the humans' hands to manipulate and employ in its exact template. The compound UA possess a wide range of biological potential but this is not the final shape of UA to be employed. It should be transformed with various angles to get an appropriate template for a specific diseased condition. A myriad of compounds have been reported to possess negligible

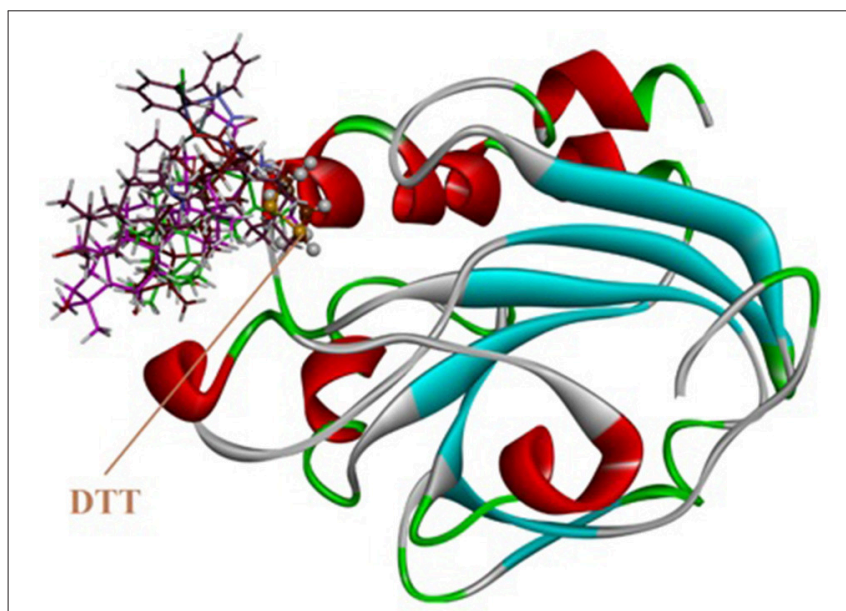

FIGURE 9 | Superposed 3D modeled diagram of some metal complexes into the active site of $3 \mathrm{MNG}$. activities alone while in complex form their activities have been dramatically enhanced due to the action of various metals and other functional groups (Bruijnincx and Sadler, 2008). No doubt, the metals present in our body are extremely important in the terms of various metabolic processes in our body (Zatta et al., 2003). Similarly, if the activity of a nucleus enhances with the addition of certain metals then the same tactics should be applied to number of compounds so that no nucleus and no complex go unexplored. A similar attempt has been followed in the current study which has been proved to be a triumph by the results of biological activities. Only two biological activities were found out i.e., antibacterial and antioxidant, which are considered to be significant in comparison with the parent compound. These complexes are further under investigations in our labs for various biological potentials including, in-vivo, in-vitro, and in-silico studies. But the current research project may be a milestone the rest of the studies.

The metal complexes are potential for biological activities (Tarafder et al., 2001). The metal complexes of various compounds have been evaluated for different activities, especially as antimicrobials (Chohan et al., 2004; Daniel et al., 2008). Bravo and co-workers have shown that the metal complexes of quercetin possess strong antibacterial activity (Bravo and Anacona, 2001). The metal complexes of coumarins and its derivatives are potential agents against various bacterial and fungal strains (Rehman et al., 2005). The tin complexes of different compounds are potential complexes in the management of leishmaniasis, free radicals scavenging, DNA interaction and as antimicrobial (Raychaudhury et al., 2005; Raza et al., 2016). The complexes of antimony have also a vital role in antimicrobial, antioxidant and anticancer activities (Viswanathan et al., 2000; Ejidike and Ajibade, 2015; Neelofar et al., 2016). The zinc

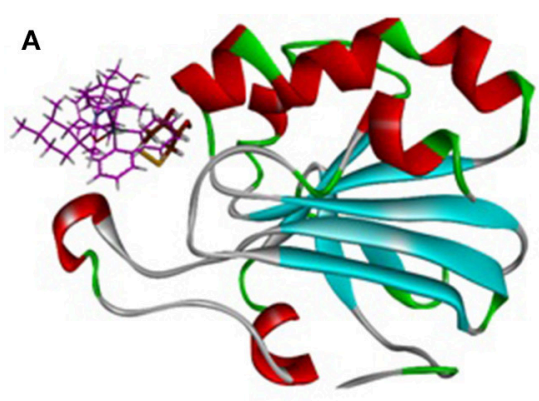

B
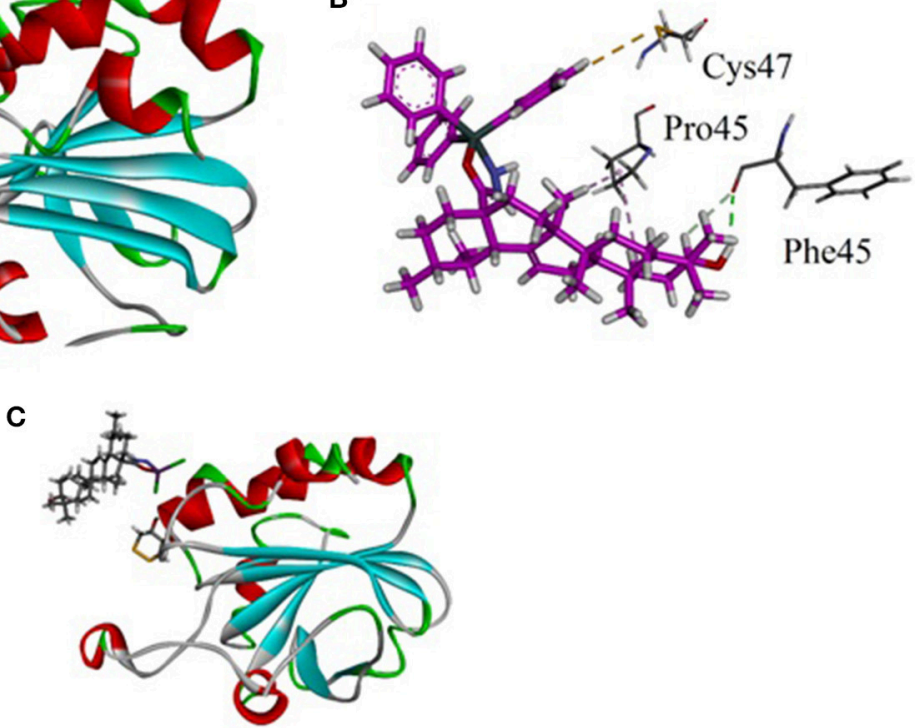

FIGURE 10 | (A) Lowest energy bonding pose of LTP superposed on DTT into the binding site of 3MNG; (B) Close-up view showing molecular interactions exhibited by LTP (purple); (C) The binding mode of LSC exhibited poor antioxidant activity. 

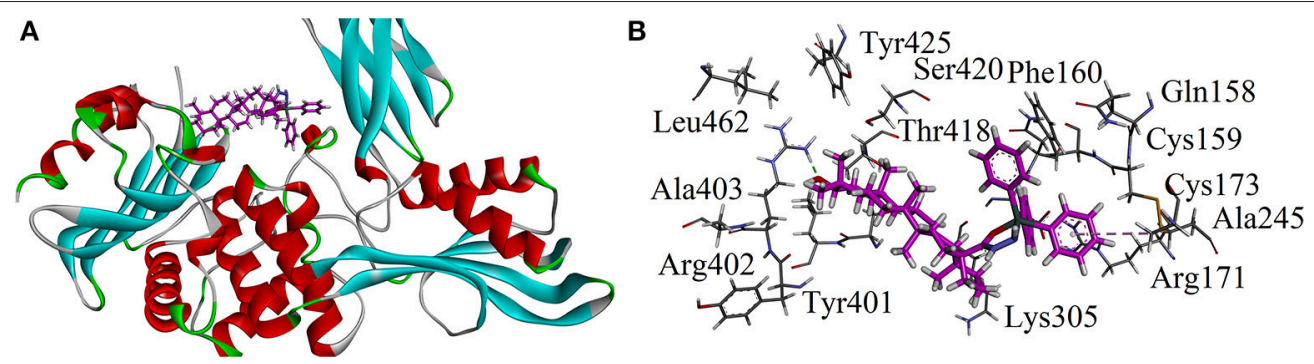

FIGURE 11 | (A) The three dimensional (3D) of triphenyl tin complex (LTP) into the binding site of 2EX9; (B) Close-up depiction of docking pose of LTP showing interactions.

complexes of several different classes of compounds have been extensively studied as an antioxidant and antimicrobial agents (Howard et al., 1973; Wang et al., 2006; Kadhum et al., 2011; Zaidi et al., 2012; Sheikh et al., 2013; Tarushi et al., 2013; Urquiza et al., 2013; Wu et al., 2013; Montazerozohori et al., 2014; Ebrahimiasl et al., 2015). As compared to other metal complexes, our synthesized derivative and its metal complexes have shown prominent antioxidant and antibacterial activities (Kadhum et al., 2011; Sadiq et al., 2017).

\section{Docking Studies \\ Docking Studies on Antioxidant Enzyme Peroxiredoxins (Prxs)}

In current study, antioxidant activity was determined by $\mathrm{DPPH}$ free radical method. However, we tried to interpret the antioxidant results at molecular level. Antioxidant assay revealed that that triphenyl tin complex exhibited significant activity. Here, we tried to interpret the experimental results with the binding mode comparison of the synthesized compounds by carrying out the docking studies against human antioxidant enzyme. We have selected peroxiredoxins (Prxs) for the interpretation of our results. Peroxiredoxins (Prxs) are thiol dependent antioxidant enzymes that reduce hydrogen peroxide and alkyl peroxides to water and alcohol (Hall et al., 2010).

Molecular docking studies were carried out on using Autodock 4.2. The three dimensional structure of human PrxV (PDB code $3 \mathrm{MNG}$ ) with a competitive antioxidant inhibitor 1,2-dithiane-4,5-diol (DTT) was retrieved from Protein Data Bank (PDB). The binding orientation of the co-crystallized ligand is shown in Figure 8A. The active site of the enzyme consists of Pro40, Gly41, Thr44, Pro45, Gly46, Cys47, Leu116, Phe120, Arg127, and Thr147 (Figure 8B). Cys47 is considered as an essential residue for binding (Hall et al., 2010). DTT established four strong hydrogen bonding interactions. A bifurcated hydrogen bond is established between two hydroxyl groups of DTT and Gly46. Thr44 and Cys47 also forms hydrogen bonding interactions with DTT hydroxyl groups (Figure 8C).

The synthesized metal complexes were docked into the active site of 3MNG. Superposed 3D modeled diagram of some metal complexes is shown in Figure 10. Figure 9 showed that not all he complexes are bounded into the binding site of the competitive inhibitor DTT.
As described above triphenyl tin complex (LTP) showed highest antioxidant activity. The computer generated 3D modeled docked diagram of the LTP showed that it is bounded into the DTT binding site (Figure 10A). Furthermore, it also shows $\pi$-sulfur interactions with catalytically important residue Cys47. A hydrogen bonding interaction was also shown by LTP and Phe45. Some hydrophobic interactions were also observed between the complex and Pro45 (Figure 10B). The estimated Free Energy of Binding of the compound is $-4.34 \mathrm{kcal} / \mathrm{mol}$. Antimony trichloride complex (LSC) was found least active complex with antioxidant activity zero at $125 \mu \mathrm{g} / \mathrm{ml}$. The binding mode of LSC is shown in Figure 10C. It is revealed from Figure 10C that LSC bounded away from DTT and have shown no interactions with catalytically important amino acid residues.

\section{Docking Studies on Antibacterial Target Protein Binding Penicillin}

Bacterial cell wall is a validated target for the drug discovery researchers because the enzyme involved in its synthesis has no counterpart in mammalians. Penicillin antibiotics area major class that targets the bacterial cell wall (Sadiq et al., 2017). The standard drug used in our antibiotic study was penicillin $\mathrm{V}$ also known as phenoxymethylpenicillin. Penicillin V, like other penicillin derivatives, inhibits the cell wall peptidoglycan.

Keeping in view the mechanism of action of standard drug used, we carried out docking studies on penicillin binding protein (PBP). Crystal structure of PBP in complex with penicillin V was retrieved from Protein Data Bank (PDB code 2EX9). The three dimensional (3D) of triphenyl tin complex (LTP) is shown in Figure 11A. The important amino acid residues are shown in Figure 11B. Compound LTP showed one hydrogen binding interactions with Arg402 and an hydrophobic interaction with Ala245. The estimated Free Energy of Binding of the LTP is -3.98 $\mathrm{kcal} / \mathrm{mol}$.

\section{CONCLUSION}

Based on the findings of our current studies, it may be inferred that the activity of ursolic acid can be enhanced by making complexes with various metals. It may also be deduced that certain metal complexes of ursolic acids have been verified as strong antibacterial and antioxidant than ursolic acid alone, which should be further verified and screened for other assays 
so that they may be proved for specific potential, which can open the way to the market. Binding mode analysis has shown a fair correlation between experimental study and estimated energy of binding.

\section{AUTHOR CONTRIBUTIONS}

MJ and KS carried out the experimental work. SA and MJ drafted the manuscript. UR performed the docking studies. AS refined the manuscript.

\section{REFERENCES}

Ahmad, S., Ullah, F., Ayaz, M., Sadiq, A., and Imran, M. (2015). Antioxidant and anticholinesterase investigations of Rumex hastatus D. Don: potential effectiveness in oxidative stress and neurological disorders. Biol. Res. 48:20. doi: 10.1186/s40659-015-0010-2

Akbas, E., and Berber, I. (2005). Antibacterial and antifungal activities of new pyrazolo [3, 4-d] pyridazin derivatives. Eur. J. Med. Chem. 40, 401-405. doi: 10.1016/j.ejmech.2004.12.001

Audrieth, L., Scott, E. S., and Kippur, P. S. (1954). Hydrazine derivatives of the carbonic and thiocarbonic acids. I. The preparation and properties of thiocarbohydrazide. J. Organ. Chem. 19, 733-741. doi: 10.1021/jo01370a006

Balanehru, S., and Nagarajan, B. (1992). Intervention of adriamycin induced free radical damage. Biochem. Int. 28, 735-744.

Baricevic, D., Sosa, S., Della Loggia, R., Tubaro, A., Simonovska, B., Krasna, A., et al. (2001). Topical anti-inflammatory activity of Salvia officinalis L. leaves: the relevance of ursolic acid. J. Ethnopharmacol. 75, 125-132. doi: 10.1016/S0378-8741(00)00396-2

Belkheiri, N., Bouguerne, B., Bedos-Belval, F., Duran, H., Bernis, C., Salvayre, R., et al. (2010). Synthesis and antioxidant activity evaluation of a syringic hydrazones family. Eur. J. Med. Chem. 45, 3019-3026. doi: 10.1016/j.ejmech.2010.03.031

Blunden S. J., and Evans C. J. (1990). "Organotin Compounds," in Anthropogenic Compounds. The Handbook of Environmental Chemistry, Vol. 3/3E, ed O. Hutzinger (Berlin; Heidelberg: Springer), 1-44.

Bravo, A., and Anacona, J. R. (2001). Metal complexes of the flavonoid quercetin: antibacterial properties. Transit. Met. Chem. 26, 20-23. doi: 10.1023/A:1007128325639

Bruijnincx, P. C., and Sadler, P. J. (2008). New trends for metal complexes with anticancer activity. Curr. Opin. Chem. Biol. 12, 197-206. doi: 10.1016/j.cbpa.2007.11.013

Chohan, Z. H., Pervez, H., Rauf, A., Khan, K. M., and Supuran, C. T. (2004). Isatin-derived antibacterial and antifungal compounds and their transition metal complexes. J. Enzyme Inhib. Med. Chem. 19, 417-423. doi: 10.1080/14756360410001710383

Atta-ur-Rahman, Choudhary, M. I., and Thomsen, W. J. (2001). Bioassay Techniques for Drug Development. Reading: Taylor \& Francis Harwood Academic Publishers.

Daniel, V. P., Murukan, B., Kumari, B. S., and Mohanan, K. (2008). Synthesis, spectroscopic characterization, electrochemical behaviour, reactivity and antibacterial activity of some transition metal complexes with 2-(Nsalicylideneamino)-3-carboxyethyl-4, 5-dimethylthiophene. Spectrochimi. Acta A Mol. Biomol. Spectrosc. 70, 403-410. doi: 10.1016/j.saa.2007.11.003

Davies, A. G., and Smith, P. J. (1980). Recent advances in organotin chemistry. Adv. Inorg. Chem. Radiochem. 23, 1-77. doi: 10.1016/S0065-2792(08)60091-6

do Nascimento, P. G., Lemos, T. L., Bizerra, A., Arriaga, Â., Ferreira, D. A., Santiago, G. M., et al. (2014). Antibacterial and antioxidant activities of ursolic acid and derivatives. Molecules 19, 1317-1327. doi: 10.3390/molecules19011317

Easmon, J., Pürstinger, G., Heinisch, G., Roth, T., Fiebig, H. H., Holzer, W., et al. (2001). Synthesis, cytotoxicity, and antitumor activity of copper (II) and iron (II) complexes of $4 \mathrm{~N}$-azabicyclo [3.2.2] nonane thiosemicarbazones derived from acyl diazines. J. Med. Chem. 44, 2164-2171. doi: 10.1021/jm000979z

Ebrahimiasl, S., Zakaria, A., Kassim, A., and Basri, S. N. (2015). Novel conductive polypyrrole/zinc oxide/chitosan bionanocomposite: synthesis,

\section{FUNDING}

This research received no specific grant from any funding agency in the public, commercial, or not-for-profit sectors.

\section{ACKNOWLEDGMENTS}

We are thankful to the Riphah International University, Islamabad for providing the space to carry out the whole research work in a placid environment.

characterization, antioxidant, and antibacterial activities. Int. J. Nanomedicine 10, 217-227. doi: 10.2147/IJN.S69740

Ejidike, I. P., and Ajibade, P. A. (2015). Transition metal complexes of symmetrical and asymmetrical Schiff bases as antibacterial, antifungal, antioxidant, and anticancer agents: progress and prospects. Rev. Inorgan. Chem. 35, 191-224. doi: 10.1515/revic-2015-0007

Ergene, E., Sivas, H., and Benkli, K. (2010). Biological activities of Cu (II) and Hg (II) complexes of a heptadentate Schiff base ligand. Turk. J. Biol. 34, 379-387. doi: 10.3906/biy-0901-17

Farina, C., Pinza, M., and Pifferi, G. (1998). Synthesis and anti-ulcer activity of new derivatives of glycyrrhetic, oleanolic and ursolic acids. Farmaco 53, 22-32. doi: 10.1016/S0014-827X(97)00013-X

Furtado, R. A., Rodrigues, É. P., Araújo, F. R., Oliveira, W. L., Furtado, M. A., Castro, M. B., et al. (2008). Ursolic acid and oleanolic acid suppress preneoplastic lesions induced by 1, 2-dimethylhydrazine in rat colon. Toxicol. Pathol. 36, 576-580. doi: 10.1177/0192623308317423

Greenwood, N. N., and Earnshaw, A. (2012). Chemistry of the Elements. Oxford: Elsevier Butterworth Heinemann.

Gum, R. J., Gaede, L. L., Koterski, S. L., Heindel, M., Clampit, J. E., Zinker, B. A., et al. (2003). Reduction of protein tyrosine phosphatase 1B increases insulin-dependent signaling in ob/ob mice. Diabetes 52, 21-28. doi: $10.2337 /$ diabetes.52.1.21

Gürkök, G., Coban, T., and Suzen, S. (2009). Melatonin analogue new indole hydrazide/hydrazone derivatives with antioxidant behavior: synthesis and structure-activity relationships. J. Enzyme Inhib. Med. Chem. 24, 506-515. doi: 10.1080/14756360802218516

Harle, H. D., Ingram, J. A., Leber, P. A., Hess, K. R., and Yoder, C. H. (2003). A simple method for determination of solubility win the first-year laboratory. J. Chem. Educ. 80:560. doi: 10.1021/ed080p560

Hall, A., Nelson, K. J., Poole, L. B., and Karplus, P. A. (2010). Structure-based insights into the catalytic power and conformational dexterity of peroxiredoxins. Antioxid. Redox Signal. 15, 795-815. doi: 10.1089/ars.2010.3624

Howard, J., Ohkatsu, Y., Chenier, J., and Ingold, K. (1973). Metal complexes as antioxidants. I. The reaction of zinc dialkyldithiophosphates and related compounds with peroxy radicals. Can. J. Chem. 51, 1543-1553. doi: $10.1139 / \mathrm{v} 73-235$

Irobi, O., Moo-Young, M., and Anderson, W. (1996). Antimicrobial activity of Annatto (Bixa orellana) extract. Int. J. Pharmacog. 34, 87-90. doi: 10.1076/phbi.34.2.87.13201

Jastrzebski, J. T., and Van Koten, G. (1993). Intramolecular coordination in organotin chemistry. Adv. Organomet. Chem. 35, 241-294. doi: 10.1016/S0065-3055(08)60493-1

Kadhum, A. A., Mohamad, A. B., Al-Amiery, A. A., and Takriff, M. S. (2011). Antimicrobial and antioxidant activities of new metal complexes derived from 3-aminocoumarin. Molecules 16, 6969-6984. doi: 10.3390/molecules16086969

Kalani, K., K., Yadav, D., Singh, A., Khan, F., Godbole, M. M., and Srivastava, S. K. (2014). QSAR guided semi-synthesis and in-vitro validation of anticancer activity in ursolic acid derivatives. Curr. Top. Med. Chem. 14, 1005-1013. doi: 10.2174/1568026614666140324121606

Kim, Y. C., Karton, Y., Ji, X. D., Melman, N., Linden, J., and Jacobson, K. A. (1999). Acyl-hydrazide derivatives of a xanthine carboxylic congener (XCC) as selective antagonists at human A2B adenosine receptors. Drug Dev. Res. 47, 178-188. doi: 10.1002/(SICI)1098-2299(199908)47:4\&lt;178::AID-DDR4\&gt;3.0.CO;2-L 
Leplège, A., and Hunt, S. (1997). The problem of quality of life in medicine. JAMA 278, 47-50. doi: 10.1001/jama.1997.03550010061041

Liu, J. (1995). Pharmacology of oleanolic acid and ursolic acid. J. Ethnopharmacol. 49, 57-68. doi: 10.1016/0378-8741(95)90032-2

Liu, J. (2005). Oleanolic acid and ursolic acid: research perspectives. J. Ethnopharmacol. 100, 92-94. doi: 10.1016/j.jep.2005.05.024

Montazerozohori, M., Yadegari, S., Naghiha, A., and Veyseh, S. (2014). Synthesis, characterization, electrochemical behavior, thermal study and antibacterial/antifungal properties of some new zinc (II) coordination compounds. J. Ind. Eng. Chem. 20, 118-126. doi: 10.1016/j.jiec.2013.04.017

Abdu Musad, E., Mohamed, R., Saeed, B. A., Vishwanath, B. S., and Rai, K. L. (2011). Synthesis and evaluation of antioxidant and antibacterial activities of new substituted bis (1, 3, 4-oxadiazoles), 3, 5-bis (substituted) pyrazoles and isoxazoles. Bioorg. Med. Chem. Lett. 21, 3536-3540. doi: 10.1016/j.bmcl.2011.04.142

Neelofar, N., Ali, N., Ahmad, S., El-Salam, N. M. A., Ullah, R., Nawaz, R., et al. (2016). Synthesis and evaluation of antioxidant and antimicrobial activities of Schiff base tin (II) complexes. Trop. J. Pharm. Res. 15, 2693-2700. doi: 10.4314/tjpr.v15i12.22

Parodi, S., De Flora, S., Cavanna, M., Pino, A., Robbiano, L., Bennicelli, C., et al. (1981). DNA-damaging activity in vivo and bacterial mutagenicity of sixteen hydrazine derivatives as related quantitatively to their carcinogenicity. Cancer Res. 41, 1469-1482.

Raychaudhury, B., Banerjee, S., Gupta, S., Singh, R. V., and Datta, S. C. (2005). Antiparasitic activity of a triphenyl tin complex against Leishmania donovani. Acta Trop. 95, 1-8. doi: 10.1016/j.actatropica.2005.03.008

Raza, A., Xu, X., Xia, L., Xia, C., Tang, J., and Ouyang, Z. (2016). Quercetiniron complex: synthesis, characterization, antioxidant, DNA binding, DNA cleavage, and antibacterial activity studies. J. Fluoresc. 26, 2023-2031. doi: 10.1007/s10895-016-1896-y

Rehman, S. U., Chohan, Z. H., Gulnaz, F., and Supuran, C. T. (2005). Invitro antibacterial, antifungal and cytotoxic activities of some coumarins and their metal complexes. J. Enzyme Inhib. Med. Chem. 20, 333-340. doi: 10.1080/14756360500141911

Robitzek, E. H., Selikoff, I. J., and Ornstein, G. G. (1952). Chemotherapy of human tuberculosis with hydrazine derivatives of isonicotinic acid. (preliminary report of representative cases.). Q. Bull. Sea View Hosp. 13, 27-51.

Sadiq, A., Ahmad, S., Ali, R., Ahmad, F., Ahmad, S., Zeb, A., et al. (2016). Antibacterial and antifungal potentials of the solvents extracts from Eryngium caeruleum, Notholirion thomsonianum and Allium consanguineum. BMC Complement. Altern. Med. 16:478. doi: 10.1186/s12906-016-1465-6

Sadiq, A., Mahmood, F., Jan, M. S., Ahmad, S., Rashid, U., Ayaz, M., et al. (2017). Ethyl 3-oxo-2-(2, 5-dioxopyrrolidin-3-yl) butanoate derivatives: Anthelmintic and cytotoxic potentials, antimicrobial and docking studies. Front. Chem. 5:119. doi: 10.3389/fchem.2017.00119

Sadiq, A., Mahmood, F., Ullah, F., Ayaz, M., Ahmad, S., Haq, F. U., et al. (2015). Synthesis, anticholinesterase and antioxidant potentials of ketoesters derivatives of succinimides: a possible role in the management of Alzheimer's. Chem. Cent. J. 9:31. doi: 10.1186/s13065-015-0107-2.

Salimon, J., Salih, N., Hameed, A., Ibraheem, H., and Yousif, E. (2010). Synthesis and antibacterial activity of some new 1,3,4-oxadiazole and 1, 3, 4-thiadiazole derivatives. J. Appl. Sci. Res. 6, 866-870.

Selikoff, I. J., Robitzek, E. H., and Ornstein, G. G. (1952). Toxicity of Hydrazine derivatives of isonicotinic acid in the chemotherapy of human tuberculosis. (a preliminary report.). Q. Bull. Sea View Hosp. 13, 17-26.

Shah, S. M., Shah, S. M. M., Ahmad, Z., Yaseen, M., Shah, R., Sadiq, A., et al. (2015). Phytochemicals, in vitro antioxidant, total phenolic contents and phytotoxic activity of Cornus macrophylla Wall bark collected from the North-West of Pakistan. Pak. J. Pharm. Sci. 28, 23-28.

Sheikh, J., Juneja, H., Ingle, V., Ali, P., and Hadda, T. B. (2013). Synthesis and in vitro biology of Co (II), Ni (II), Cu (II) and Zinc (II) complexes of functionalized beta-diketone bearing energy buried potential antibacterial and antiviral O, O pharmacophore sites. J. Saudi Chem. Soc. 17, 269-276. doi: 10.1016/j.jscs.2011.04.004

Shin, K. M., Kim, R. K., Azefack, T. L., David, L., Luc, S. B., Choudhary, M. I., et al. (2004). In vitro anti-inflammatory activity of 23 -hydroxyursolic acid isolated from Cussonia bancoensis in murine macrophage RAW 264.7 cells. Planta Med. 70, 803-807. doi: 10.1055/s-2004-827226
Sönmez, M., Berber, I., and Akbaş, E. (2006). Synthesis, antibacterial and antifungal activity of some new pyridazinone metal complexes. Eur. J. Med. Chem. 41, 101-105. doi: 10.1016/j.ejmech.2005.10.003

Tarafder, M., Kasbollah, A., Crouse, K., Ali, A., Yamin, B. M., and Fun, H.-K. (2001). Synthesis and characterization of $\mathrm{Zn}$ (II) and Cd (II) complexes of Sbenzyl- $\beta$-N-(2-pyridyl) methylenedithiocarbazate (HNNS): bioactivity of the HNNS Schiff base and its $\mathrm{Zn}$ (II), $\mathrm{Cu}$ (II) and Cd (II) complexes and the $\mathrm{X}$-ray structure of the [Zn (NNS) 2] complex. Polyhedron 20, 2363-2370. doi: 10.1016/S0277-5387(01)00817-8

Tarushi, A., Kljun, J., Turel, I., Pantazaki, A. A., Psomas, G., and Kessissoglou, D. P. (2013). Zinc (II) complexes with the quinolone antibacterial drug flumequine: structure, DNA-and albumin-binding. New J. Chem. 37, 342-355. doi: $10.1039 / \mathrm{C} 2 \mathrm{NJ} 40798 \mathrm{~A}$

Urquiza, N. M., Islas, M. S., Dittler, M. L., Moyano, M. A., Manca, S. G., Lezama, L., et al. (2013). Inhibition behavior on alkaline phosphatase activity, antibacterial and antioxidant activities of ternary methimazole-phenanthroline-copper (II) complex. Inorganica Chim. Acta 405, 243-251. doi: 10.1016/j.ica.2013.05.022

Viswanathan, P., Sriram, V., and Yogeeswaran, G. (2000). Sensitive spectrophotometric assay for 3-hydroxy-substituted flavonoids, based on their binding with molybdenum, antimony, or bismuth. J. Agric. Food Chem. 48, 2802-2806. doi: 10.1021/jf990357q

Wang, H.-L., Yang, Z.-Y., and Wang, B.-D. (2006). Synthesis, characterization and the antioxidative activity of copper (II), zinc (II) and nickel (II) complexes with naringenin. Trans. Metal Chem. 31, 470-474. doi: 10.1007/s11243-006-0015-3

Wolska, K., Grudniak, A., Fiecek, B., Kraczkiewicz-Dowjat, A., and Kurek, A. (2010). Antibacterial activity of oleanolic and ursolic acids and their derivatives. Open Life Sci. 5, 543-553. doi: 10.2478/s11535-010-0045-x

Wu, S., Du, Y., Hu, Y., Shi, X., and Zhang, L. (2013). Antioxidant and antimicrobial activity of xylan-chitooligomer-zinc complex. Food Chem. 138, 1312-1319. doi: 10.1016/j.foodchem.2012.10.118

Yilmaz, A. D., Coban, T., and Suzen, S. (2012). Synthesis and antioxidant activity evaluations of melatonin-based analogue indolehydrazide/hydrazone derivatives. J. Enzyme Inhib. Med. Chem. 27, 428-436. doi: $10.3109 / 14756366.2011 .594048$

Yousefi, M., Tavakolinia, F., and Hassanzadeh, S. M. (2011). "Comparison of antibacterial activities of di-and tri-tin (IV) carboxylate complexes," in International Conference on Biology, Environment and Chemistry (Singapoore).

Zaidi, M. I., Wattoo, F. H., Wattoo, M. H. S., Tirmizi, S. A., and Salman, S. (2012). Antibacterial activities of nicotine and its zinc complex. Afr. J. Microbiol. Res. 6, 5134-5137. doi: 10.5897/AJMR11.1209

Zatta, P., Lucchini, R., Van Rensburg, S. J., and Taylor, A. (2003). The role of metals in neurodegenerative processes: aluminum, manganese, and zinc. Brain Res. Bull. 62, 15-28. doi: 10.1016/S0361-9230(03)00182-5

Zeb, A., Ullah, F., Ayaz, M., Ahmad, S., and Sadiq, A. (2017). Demonstration of biological activities of extracts from Isodon rugosus Wall. Ex Benth: Separation and identification of bioactive phytoconstituents by GC-MS analysis in the ethyl acetate extract. BMC Complement. Altern. Med. 17:284. doi: 10.1186/s12906-017-1798-9.

Zhong, Z., Zhong, Z., Xing, R., Li, P., and Mo, G. (2010). The preparation and antioxidant activity of 2-[phenylhydrazine (or hydrazine)-thiosemicarbazone]chitosan. Int. J. Biol. Macromol. 47, 93-97. doi: 10.1016/j.ijbiomac.2010.05.016

Ziegler, H. L., Franzyk, H., Sairafianpour, M., Tabatabai, M., Tehrani, M. D., Bagherzadeh, K., et al. (2004). Erythrocyte membrane modifying agents and the inhibition of Plasmodium falciparum growth: structure-activity relationships for betulinic acid analogues. Bioorg. Med. Chem. 12, 119-127. doi: 10.1016/j.bmc.2003.10.010

Conflict of Interest Statement: The authors declare that the research was conducted in the absence of any commercial or financial relationships that could be construed as a potential conflict of interest.

Copyright $(2018$ Jabeen, Ahmad, Shahid, Sadiq and Rashid. This is an open-access article distributed under the terms of the Creative Commons Attribution License (CC $B Y)$. The use, distribution or reproduction in other forums is permitted, provided the original author(s) and the copyright owner are credited and that the original publication in this journal is cited, in accordance with accepted academic practice. No use, distribution or reproduction is permitted which does not comply with these terms. 